\title{
Do novel genes drive morphological novelty? An investigation of the nematosomes in the sea anemone Nematostella vectensis
}

\author{
Leslie S. Babonis ${ }^{1 *}$, Mark Q. Martindale ${ }^{1,2}$ and Joseph F. Ryan ${ }^{1,2}$
}

\begin{abstract}
Background: The evolution of novel genes is thought to be a critical component of morphological innovation but few studies have explicitly examined the contribution of novel genes to the evolution of novel tissues. Nematosomes, the free-floating cellular masses that circulate through the body cavity of the sea anemone Nematostella vectensis, are the defining apomorphy of the genus Nematostella and are a useful model for understanding the evolution of novel tissues. Although many hypotheses have been proposed, the function of nematosomes is unknown. To gain insight into their putative function and to test hypotheses about the role of lineage-specific genes in the evolution of novel structures, we have re-examined the cellular and molecular biology of nematosomes.

Results: Using behavioral assays, we demonstrate that nematosomes are capable of immobilizing live brine shrimp (Artemia salina) by discharging their abundant cnidocytes. Additionally, the ability of nematosomes to engulf fluorescently labeled bacteria (E. coli) reveals the presence of phagocytes in this tissue. Using RNA-Seq, we show that the gene expression profile of nematosomes is distinct from that of the tentacles and the mesenteries (their tissue of origin) and, further, that nematosomes (a Nematostella-specific tissue) are enriched in Nematostella-specific genes.

Conclusions: Despite the small number of cell types they contain, nematosomes are distinct among tissues, both functionally and molecularly. We provide the first evidence that nematosomes comprise part of the innate immune system in N. vectensis, and suggest that this tissue is potentially an important place to look for genes associated with pathogen stress. Finally, we demonstrate that Nematostella-specific genes comprise a significant proportion of the differentially expressed genes in all three of the tissues we examined and may play an important role in novel cell functions.
\end{abstract}

Keywords: Novelty, Cnidocyte, Nematosome, Immune, Phagocytosis, RNA-Seq, Nematostella vectensis,

Taxonomically-restricted genes

\section{Background}

Understanding the evolution of animal complexity requires an understanding of how new traits arise. Studies of novel trait evolution typically invoke either the origin of new genes $[1,2]$ or the evolution of new relationships among conserved genes [3] as the primary drivers of evolutionary innovation. Collectively, these studies

\footnotetext{
* Correspondence: babonis@whitney.ufl.edu

'Whitney Laboratory for Marine Bioscience, University of Florida, 9505 Ocean

Shore Blvd, St. Augustine, FL 32080, USA

Full list of author information is available at the end of the article
}

suggest that novel genes can be generated in several ways, including: duplication and divergence of conserved genes, RNA-mediated duplication/retroposition, exon shuffling/mis-splicing of RNA and other modifications to coding sequence, horizontal transfer of genes from other taxa, de novo evolution of an open reading frame in previously non-coding sequence, and overprinting (i.e., transcription from multiple reading frames in the same gene) $[1,2,4]$. Indeed, this diversity of mechanisms underlying the generation of new coding sequence has led to the suggestion that novel genes are generated with 
high frequency across lineages [5]. In support of this, approximately 10-20\% of the genes in all sequenced genomes (as of 2010) comprise novel/taxonomicallyrestricted genes $[6,7]$, but the putative role of these abundant novel genes in driving evolutionary innovation is far from clear.

A recent study of drosophilids demonstrated that equally large proportions of novel (Drosophila-specific) and conserved genes ( 30\% of each) were critical for viability, supporting the idea that recently evolved genes can be essential to the biology of the organism [8]. Several additional studies provide support for novel/ taxonomically-restricted genes in generating lineagespecific morphological features (e.g., [9-13]) but surprisingly, most of these studies have identified only few novel genes to be contributing to the novel morphological trait of interest. Thus, despite their focus on the role of novel genes in the evolution of a novel trait, these studies actually reveal that the majority of the genes involved in evolutionary innovation are conserved. One challenge to studies of evolutionary novelty is the identification of a truly novel tissue to use as a model $[14,15]$. Additionally, limited availability of data from closely related taxa means that many genes identified as "novel" may actually reflect sparse taxon sampling. Poor tissue sampling may pose an even bigger problem, leading to a general overestimation of the value of novel genes in the evolution of novel tissues merely because no other tissues were sampled. In fact, only few studies have explicitly examined the abundance and distribution of novel genes across cell/ tissue types $[12,16,17]$ and their results regarding the importance of novel genes have been conflicting.

Cnidarians have become a valuable model for the evolution of novelty because they possess an unequivocal example of a single-celled novelty: the cnidocyte (stinging cell). Cnidocytes are complex sensory/effector cells that exhibit significant morphological diversity across cnidarians (for a review of cnidocyte diversity, see [18]). Used primarily in prey capture and defense, cnidocytes are abundant in the tentacles, which are the primary feeding apparatus of cnidarians. Among anthozoans (e.g., corals and sea anemones), one of the notable synapomorphies is the presence of mesenteries $[19,20]$, multifunctional internal tissues of mixed developmental origin (endodermal and ectodermal) that contain gonadal and digestive cells as well as a diverse repertoire of other cell types, including cnidocytes. During feeding, the mesenteries become apposed to ingested prey items, presumably enabling the use of their cnidocytes and ectodermal gland cells to assist in immobilization/digestion of prey tissues [21]. The mesenteries of sea anemones in the superfamily Metridioidea [22] are modified to include threadlike distal extensions called "acontia" which are replete with cnidocytes. Interestingly, the suite of cnidocytes that populate the acontia can be distinct from that of the mesenteries, their tissue of origin [23]. While cnidarians may superficially appear simple, they clearly exhibit tissue-specific distributions of cell types resulting in a surprisingly high level of morphological complexity.

Nematostella vectensis (the starlet sea anemone) has emerged as an important model for diverse studies of cellular and molecular biology [24]; yet one of the most intriguing tissues in this animal remains largely unstudied: the nematosomes. First described by Stephenson in 1935 [25], nematosomes are an enigmatic free-floating tissue that circulates through the gastrovascular cavity (Additional file 1) in adult sea anemones, occasionally coming to rest along the internal surface of the body wall (Fig. 1a) [26, 27]. The presence of nematosomes in $N$. vectensis, Milne-Edwardsia polaris (later N. polaris), and Milne-Edwardsia nathorstii (later N. nathorstii), and their absence in other members of the Edwarsiidae led to the early designation of the nematosomes as the defining apomorphy of the genus Nematostella ([25, 28-30], as cited by [31,32]). Despite this, little is known about the biology of this tissue. Nematosomes are small, multicellular masses of cells comprised largely of cnidocytes (Fig. 1b) and, like the acontia of metridoidean cnidarians, nematosomes are derivatives of the mesenteries [31]. Given the critical role of cnidocytes in the feeding behavior of sea anemones, early hypotheses of nematosome function suggested a role for this novel tissue in facilitating immobilization of ingested prey [27, 32]; yet support for this hypothesis has been inconclusive [33]. Histological studies of nematosomes confirmed that this tissue lacks gland cells leading to the conclusion that nematosomes play no role in digestion [33] but provided no convincing alternative function and no further information about the cell types present in these structures. The observation of abundant nematosomes in the jelly matrix surrounding the spawned egg masses [26, 34] led to a hypothesized role in protection of the developing embryos; yet, this hypothesis, too, has received mixed support $[33,35]$. The long history of conflicting results regarding the putative function of nematosomes even led to the hypothesis that nematosomes play no role at all in the biology of $N$. vectensis [33], ultimately relegating the diagnostic morphological feature of the genus Nematostella merely to an intriguing curiosity.

Nematosomes are thought to develop continuously throughout the adult life of $N$. vectensis (albeit at a rate influenced by some undefined mechanism) making them a tractable model for understanding patterning and identity in an adult tissue. Since nematosomes are restricted only to the genus Nematostella (i.e., they have no phylogenetic homologs) and they are found only in adult polyps (i.e., they have no serial homologs), these unique 


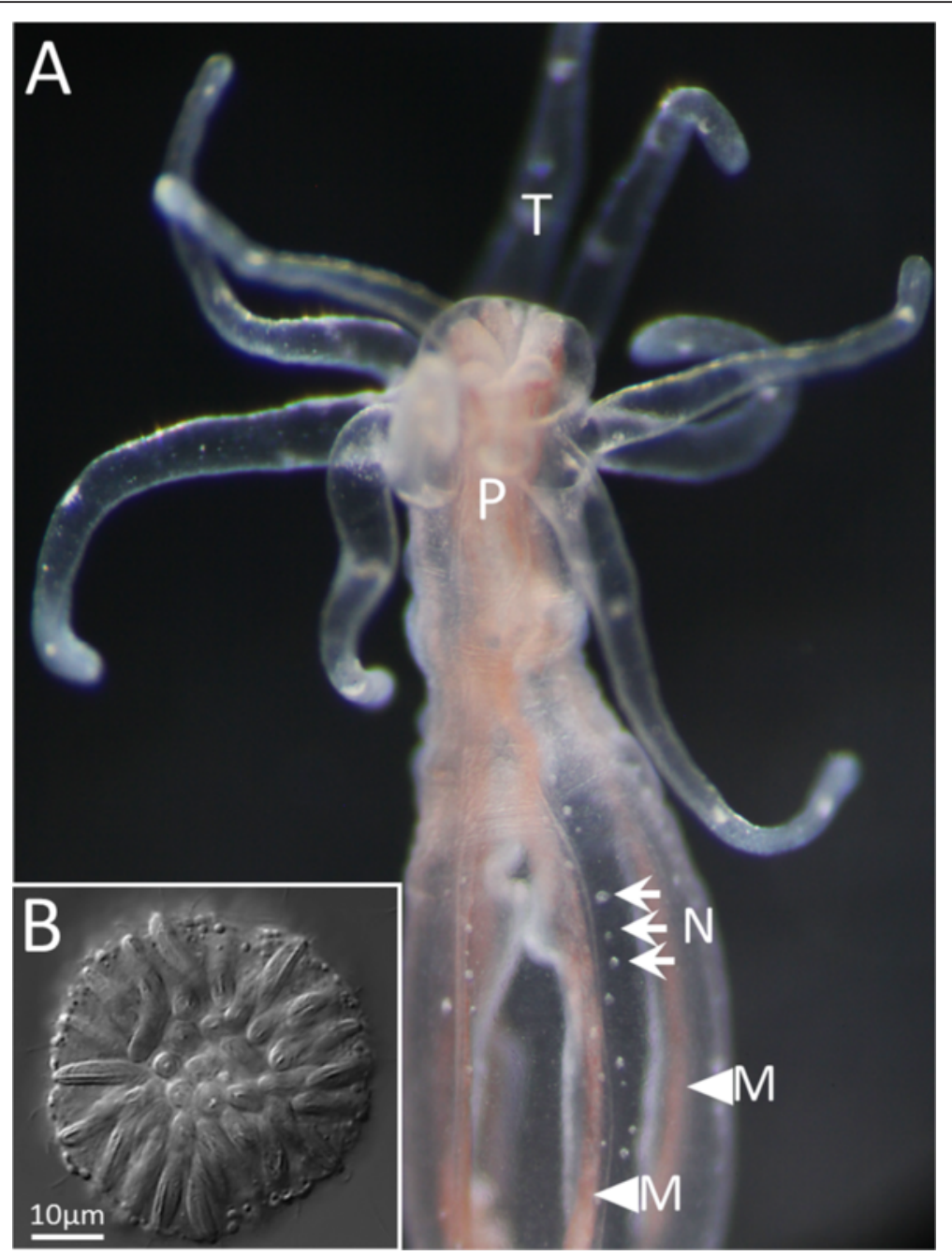

Fig. 1 Nematosomes in N. vectensis. a A live image of a young adult polyp (10-tentacle stage); several nematosomes (N, arrows) are visible at rest along the internal surface of the body wall near the insertions of the mesenteries ( $M$, arrowheads). The pharynx $(P)$ and tentacles $(T)$ are also visible. b A DIC optical section of an isolated nematosome

structures are an unequivocal example of a phenotypic/ tissue-level novelty [36, 37]. Whether this novel tissue expresses novel genes and cell types, however, is unknown. Herein, we (i) assess the function of this tissue by explicitly testing existing hypotheses from the literature, (ii) examine the morphology of this tissue for evidence of novel cell types, (iii) characterize the gene expression profile of the nematosomes (using RNA-Seq), and (iv) test hypotheses about the contribution of novel genes to novel structures by evaluating the distribution of novel and conserved genes in nematosomes and other tissues. Together, our results confirm that nematosomes comprise more than just a mass of sloughed cnidocytes and provide support for a novel role for this tissue in the innate immune system of $N$. vectensis. We further report that Nematostella-specific genes are indeed overrepresented in this Nematostella-specific tissue but these novel genes also comprise a large proportion of the upregulated transcripts across tissues, suggesting that novel genes may play important roles in the tissue-specific biology of all tissues.

\section{Results}

\section{Nematosome development}

Nematosomes first appear in the body cavity of $N$. vectensis near the onset of reproductive maturity, after development of the ciliated tracts of the mesenteries (personal observations). Although material can be observed circulating through the body cavity of small polyps before maturation (Additional file 2A), this material is largely unicellular and does not contain cnidocytes. Similar material can be seen circulating through the cavity of adult polyps (Additional file 2B) but a comparison of the size of these particles with the nematosomes that also circulate in the cavity of adults makes clear that the former are not nematosomes. As 
noted by previous studies, nematosomes form by budding from the ectodermal (cnidocyte-rich) portion of the mesenteries [31]. Here, we demonstrate that nematosomes bud from regions of the mesenteries that are already rich in mature cnidocyte capsules (Fig. 2a, b) and the presence of cells in S-phase (labeled by EdU; Fig. 2c) suggests that the process of budding involves proliferation of the cells around the mature cnidocytes. Two pieces of evidence suggest that nematosome budding occurs only after completion of cnidocyte development: first, proliferative cells cannot be detected in this tissue. Despite the many thousands of nematosomes we assayed $(N=4$ independent experiments on juvenile polyps or $N=3$ independent experiments on egg masses, each representing thousands of nematosomes per experiment), we have observed only three nematosomes with proliferative cells (Additional file 3A). These results suggest new cells (cnidocytes or any other cell types) are not being made in the nematosomes after they leave the mesentery. Second, our attempts to identify developing cnidocytes directly have all failed. Using TEM, we have identified developing cnidocytes in the tentacle ectoderm from their unusual appearance (Fig. 2d) but have never seen developing cnidocytes in thin sections from nematosomes (e.g., Figs. 5 and 6). Also, using antibodies directed against three different minicollagens (mcol 1,3 , and 4), which are known to label only developing cnidocytes [38], we demonstrate that early planula stage embryos have abundant developing cnidocytes throughout the ectoderm while nematosomes assayed at the same time with the same antibodies, do not (Fig. 2e-g). In concert with the previous observation that nematosomes appear to lack cells expressing minicollagen RNA [39], these data suggest that nematosomes are shed from distinct regions of the mesenteries only after their cellular complement has been determined.

\section{Nematosomes subdue prey}

To test the hypothesis that nematosomes are essentially non-functional waste material sloughed from the mesenteries [33], we assessed the capacity of nematosomes to subdue prey. To do this, we isolated live nematosomes from freshly spawned egg masses and concentrated them

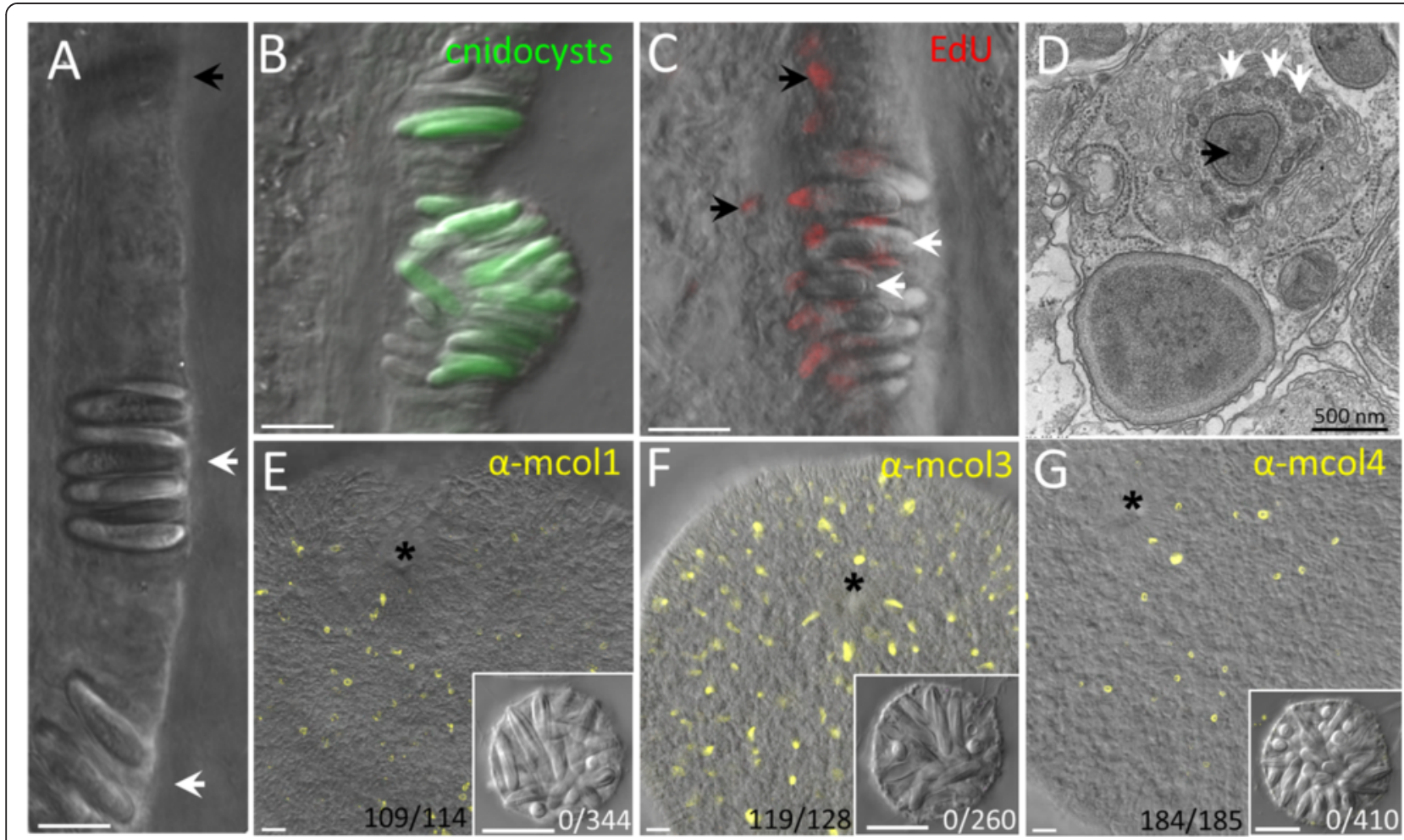

Fig. 2 Nematosome budding. a Clusters of cnidocytes (white arrows) are visible in the ectodermal mesentery before budding (the black arrow indicates a cluster in a different focal plane). b A later stage in the budding process showing the abundance of mature cnidocytes (green; $143 \mu \mathrm{M}$ $\mathrm{DAPI}$ ) as the nematosome begins to protrude from the mesentery epithelium. $\mathbf{c}$ Proliferative nuclei (red, $100 \mu \mathrm{M} \mathrm{EdU}$ ) are visible in the basal region of the epithelium, between and below the cnidocytes (white arrows). Proliferation also occurs outside of the budding zone (black arrows). $\mathbf{d}$ A developing cnidocyte from tentacle ectoderm; white arrows indicate the cnidocyst tubule which develops outside of and around the cnidocyst capsule (black arrow). e-g Immunohistochemistry performed in early planula stage embryos reveals abundant developing cnidocytes labeled with anti-mcol1, anti-mcol3, or anti-mcol4 antibodies (yellow). Nematosomes incubated at the same time in the same aliquot of each antibody lack staining (insets); the number of tissues (embryos or nematosomes) observed to have mcol ${ }^{+}$cells is indicated. The blastopore of each embryo is indicated by * for orientation. All scale bars represent $10 \mu \mathrm{m}$ unless otherwise specified 
in a small amount of $1 / 3 \mathrm{X}$ filtered seawater (FSW) in a small glass dish. When recently hatched brine shrimp (Artemia salina) were introduced into the dish with the nematosomes, the shrimp became immobilized almost immediately (Additional file 4), at a rate consistent with the rate at which these animals are subdued by the tips of the tentacles in an adult $N$. vectensis polyp (Additional file 5). By contrast, 1/3X FSW alone failed to have any adverse effect on the shrimp (Additional file 6). Highmagnification images taken at the end of the experiment reveal the presence of nematosome clusters adhering to the exoskeletons of the subdued shrimp (Fig. 3a) and at even higher magnification, discharged cnidocysts can be seen penetrating the exoskeleton (Fig. 3b). Likewise, a highly magnified image of an individual nematosome reveals the presence of multiple discharged cnidocysts (Fig. 3c). These data confirm that nematosomes possess the ability to subdue prey and that this is a result of cnidocyte firing.

\section{Nematosomes: more than just cnidocytes}

Nematosomes have previously been reported to contain two of the three cnidocyte types found in $N$. vectensis: basitrichous haplonemes ("basitrichs") and microbasic pmastigophores [26]. We find both of these cells types in abundance across nematosomes (Fig. 4a). (Only a single spirocyte has been observed among the thousands of nematosomes we examined; Additional file 3B). To determine if other cell types (beyond mature cnidocytes) might be present in nematosomes, we counted nuclei (Fig. 4b) and cnidocyst capsules (Fig. 4c) in two independent samples of nematosomes, each spanning the range of nematosome size ( $N=66$ and $N=41$ nematosomes, respectively). Both the total number of nuclei and the number of cnidocyst capsules increase linearly with size over the range of nematosomes examined
(Fig. 4d; nuclei $R^{2}=0.82$, cnidocytes $R^{2}=0.67, p<0.001$ ), but these relationships differ in slope (nuclei: 4.07, cnidocytes: 1.34). Because nematosomes do not contain developing cnidocytes (Fig. 2), these relationships suggest two things: first, all nematosomes have more nuclei than cnidocyst capsules and, therefore, must contain non-cnidocyte cells, and second, that large nematosomes contain more non-cnidocyte cells than do small nematosomes.

\section{Nematosome apical morphology is marked by abundant sensory cones}

In addition to cnidocytes, nematosomes are known to have abundant motile cilia $[25,26]$, although the nature of the cell type from which these cilia emerge has not been examined. Using fluorescence microscopy and an antibody directed against acetylated-tubulin, we confirm the presence of abundant cilia emerging from each isolated nematosome (Fig. 5a). Quantitative comparisons of cilium length (Fig. 5b) reveal the presence of two distinct types: Type I cilia are short, averaging $4.50+/-0.81 \mu \mathrm{m}$ in length (Fig. 5a, white arrowhead) while type II cilia are nearly four times as long $(19.84+/-1.71 \mu \mathrm{m}$ in length; Fig. 5a, white arrow). Double-labeling with phalloidin (to detect F-actin) indicates the presence of numerous actin-rich apical cones (Fig. 5a, black arrowhead), previously described in tentacle epithelia of $N$. vectensis [40-42]. Using field-emission scanning electron microscopy (FE-SEM), we confirm the presence of at least two types of apical cones, associated with cilia of different length (Fig. 5c): type I cones have abundant short stereocilia organized into concentric whorls (Fig. 5d, white arrow) from which a short (type I) cilium emerges [43]. By contrast, type II cones are composed of a single ring of stereocilia connected by inter-ciliary links (Fig. 5e, black arrow) from which type II cilia emerge. Both types of

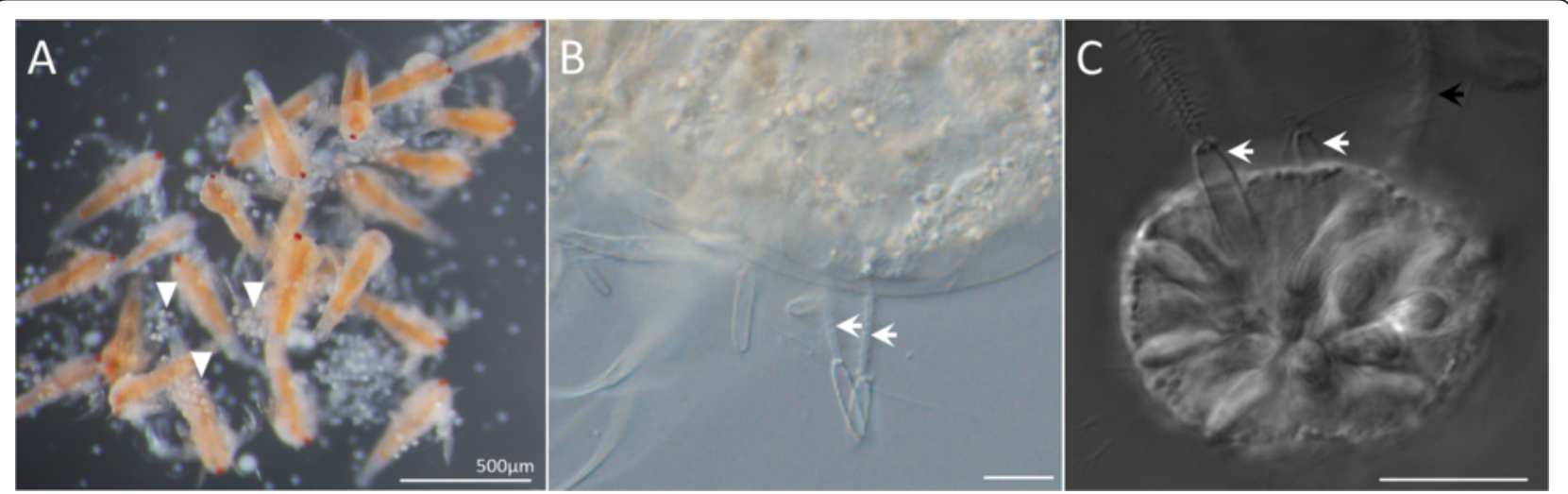

Fig. 3 Live nematosomes are capable of subduing brine shrimp (Artemia salina). (See also Additional file 4.) a Aggregates of nematosomes (arrowheads) are attached to subdued brine shrimp. b Higher magnification reveals the presence of two spent cnidocysts (arrows) protruding from the exoskeleton of the shrimp. c A high-magnification image of an isolated nematosome reveals two spent cnidocysts (white arrows). Black arrow indicates a spent cnidocyst in another focal plane. All scale bars represent $10 \mu \mathrm{m}$ unless otherwise specified 

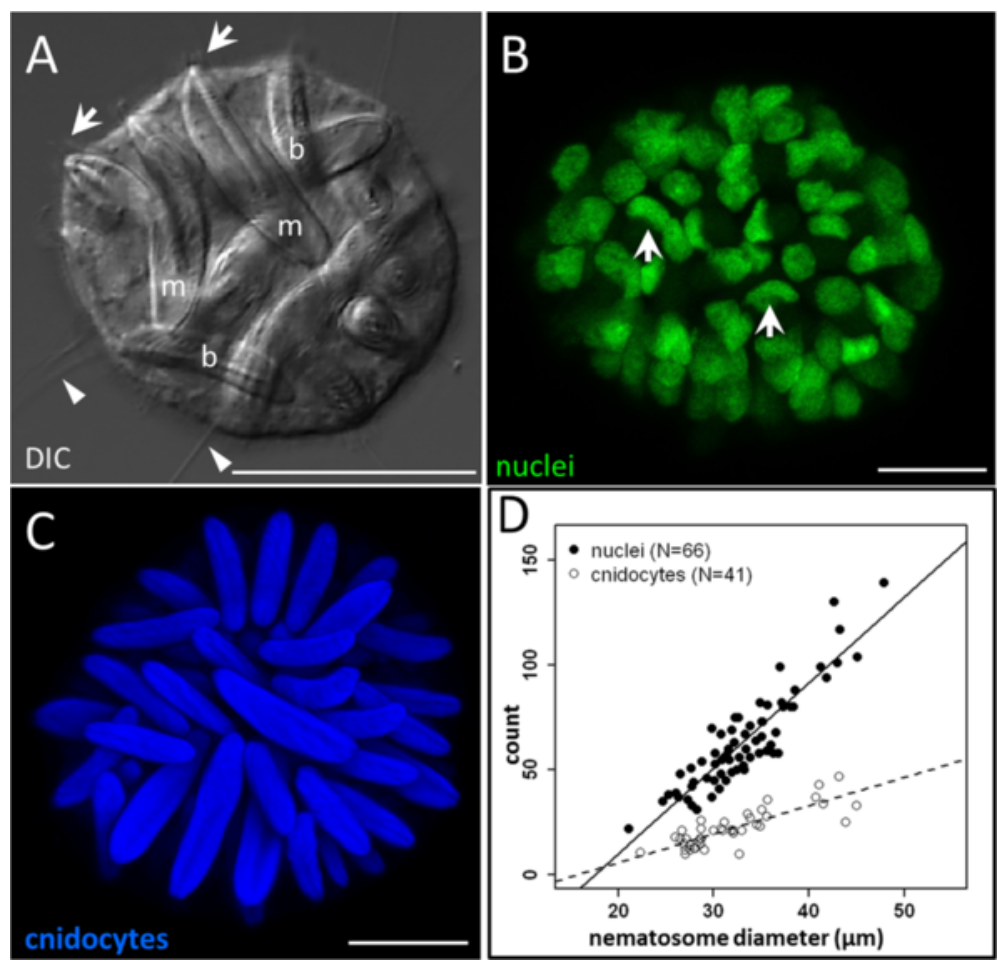

Fig. 4 Nematosomes comprise multiple cell types. a A single DIC optical section through an isolated nematosome in which two types of cnidocyte are abundant: basitrichous isorhizas (b) characterized by a thin shaft extending nearly the full length of the capsule, and microbasic-p-mastigophores (m) which have a thick shaft that extends only half the length of the capsule and has a distinctive V-shaped notch [18]. Ciliary cones are visible (arrows) at the apex of two mastigophores and several long cilia (arrowheads) can be seen emerging from the perimeter of the tissue. $\mathbf{b} \mathrm{A} 3 \mathrm{D}$ rendering of a confocal z-stack through a nematosome indicating nuclei (labeled with $1 \mu \mathrm{M}$ DAPl; green). Nuclei in cnidocytes often appear semi-lunar in shape (arrows) as they conform to the shape of the cnidocyst capsule. c A 3D rendering of a confocal z-stack through another single nematosome showing the presence of abundant mature cnidocytes (labeled with $143 \mu \mathrm{M}$ DAPl; blue). $\mathbf{d}$ The numbers of nuclei (an indicator of total cell number; closed circles) and cnidocytes (open circles) in individual nematosomes increase as a function of nematosome diameter but these relationships differ across the range of nematosomes studied (nuclei slope: 2.72, cnidocyte slope: 1.34; $p<0.001$ ANCOVA). All scale bars represent $10 \mu \mathrm{m}$

ciliary cones were abundant in nematosomes of all sizes. Thin sections indicate that type I ciliary cones are found at the apex of cnidocytes while cells with type II ciliary cones are often found adjacent to cnidocytes (Fig. 5f) in a cell type that shares morphological features with the previously described cnidocyte support cells [44].

\section{Phagocytes: a new cell identified among nematosomes}

Using TEM, we also identify an additional cell type in nematosomes with the morphology of a phagocyte. Thin sections reveal the abundance of large vacuolated cells in the periphery of an individual nematosome (Fig. 6a, black arrows). High-magnification imaging of one of these peripheral cells (Fig. 6b) reveals vesicles with the morphology of lysosomes, primary endosomes, and mature endosomes (multivesicular bodies), all within 1-2 $\mu \mathrm{m}$ of the apical membrane, consistent with the components of the endocytotic pathway in anthozoans [45]. Additionally, live nematosomes readily engulfed FITC-labeled heatinactivated E. coli (Fig. 6c) and FITC-labeled latex beads
(Fig. 6c, inset), confirming the capacity of nematosomes to phagocytose various types of particles. Together, these data provide the first evidence of phagocytotic capacity in nematosomes and suggest nematosomes may play a role in clearing the gastrovascular cavity of foreign particles.

\section{Constructing the nematosome transcriptome}

One of the advantages of working with $N$. vectensis is the availability of genomic resources, including a publicly available reference genome [46] and several independent sources of transcriptome data (e.g., $[47,48])$. However, tissue-specific transcriptome data are lacking and there have been no efforts to explicitly sequence the nematosomes. Considering the small size of the nematosomes and the fact that their abundance varies widely across individuals and throughout the year [26, 35], it is likely that this tissue was not well-represented in previous transcriptome studies. To gain insight into the molecular profile of nematosomes, we sequenced the complete set of transcripts expressed in the nematosomes and compared the 


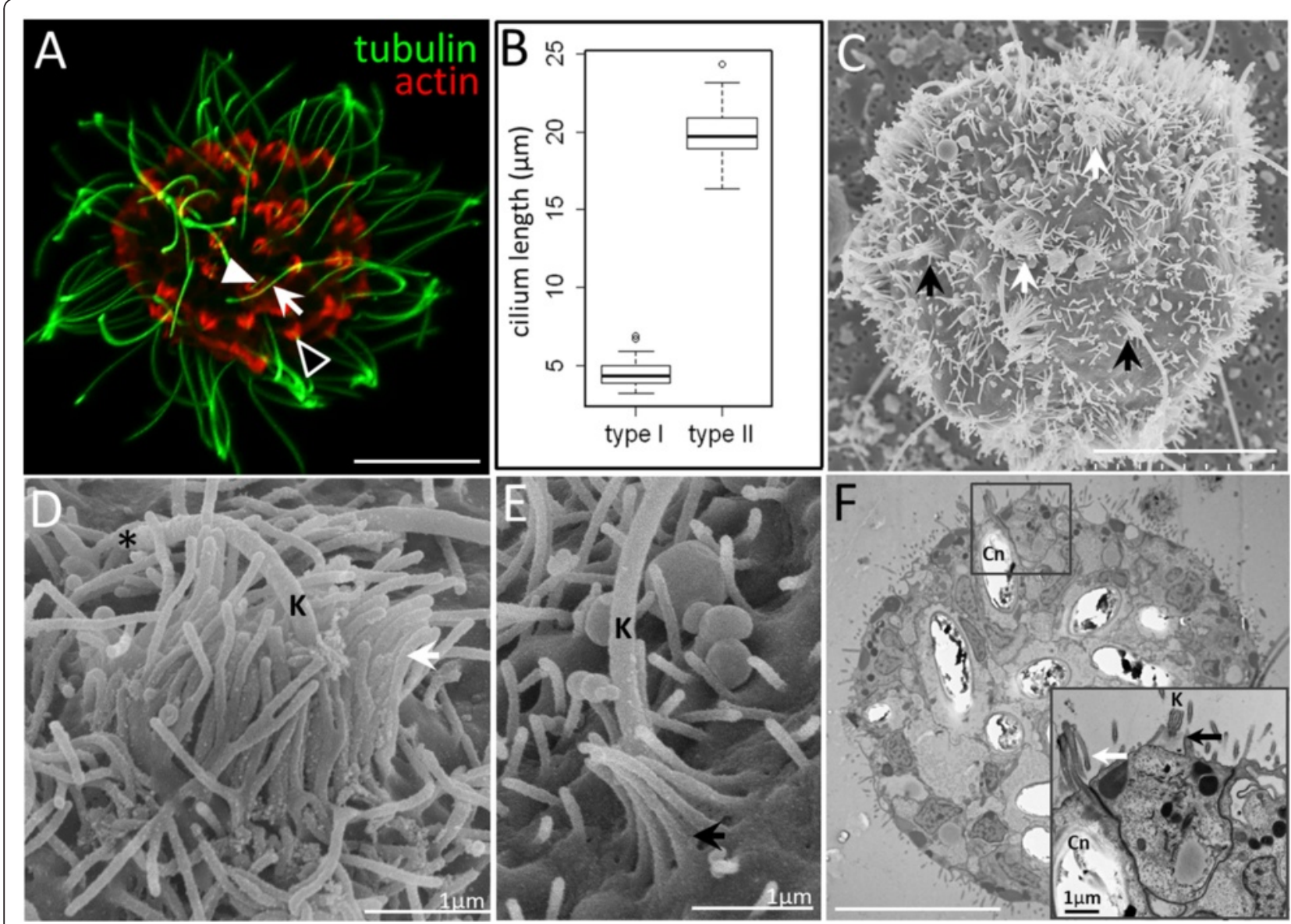

Fig. 5 Ciliary structures on the surface of a nematosome. a Double labeling of F-actin (phalloidin; red) and acetylated-tubulin (green) indicates the presence of dense actin-rich apical cones (black arrowhead) and two types of cilia: type I, short (white arrowhead) and type II, long (white arrow). b Cilium length differs significantly between type I and type II cilia (ANOVA, $p<<0.001$ ). $\mathbf{c}$ Numerous ciliary cones of different morphologies (arrows) are visible among the abundant microvilli covering the surface of a nematosome. $\mathbf{d}$ Type I ciliary cones (white arrows in A) are wide, assembled from multiple layers of stereocilia (white arrow), and surround a short central kinocilium (K; *indicates the distal tip of the cilium). e Type II ciliary cones are narrow and are composed of a single layer of stereocilia (black arrow) that surround a long kinocilium. $\mathbf{f}$ A thin section reveals the relationship of the ciliary cones and their underlying cell types. Higher magnification (inset) shows a type I ciliary cone (white arrow) at the apex of a cnidocyte (Cn) and a type II ciliary cone (black arrow) at the apex of a cell type with sub-apical vesicles of various size and electron density immediately adjacent to the cnidocyte. All scale bars represent $10 \mu \mathrm{m}$ unless otherwise specified

expression profile of this tissue with that of the mesenteries (from which nematosomes arise) and the tentacles (another cnidocyte-rich tissue).

All three tissues (nematosomes, mesenteries, and tentacles) were collected from adult polyps and sequenced in triplicate using Illumina technology, resulting in over $100 \mathrm{M}$ paired-end $100 \mathrm{bp}$ reads per tissue. In order to identify novel (i.e., Nematostella-specific) transcripts with confidence, we chose to avoid the use of published gene models (since $a b$ initio gene predictors may be biased toward identifying conserved sequences) and instead to assemble a reference transcriptome de novo, using reads from all three tissues and the de novo assembler Trinity $[49,50]$. Erroneous assembly of numerous transcript "isoforms" is a common problem with de novo assemblers and a challenge to producing reliable differential expression results [51]; to avoid interpretation of these assembly errors as novel sequences, we aimed to minimize the number of assembled transcripts. To this end, we evaluated three reference transcriptomes assembled using different methods: a full transcriptome (TR333942) was assembled from the complete set of reads from all three replicates of all three tissues trimmed for adapters and low-quality bases using Trimmomatic [52], a second transcriptome (TR118377) was assembled from all the trimmed reads from single replicate of each tissue, and a third transcriptome (NvecRef32743) was assembled from a single replicate of each tissue after trimming and subjecting reads to error-correction using AllPaths-LG [53]. All raw reads and the three transcriptomes assembled from them have been deposited in the European Nucleotide Archive, Project Accession: PRJEB13676. 


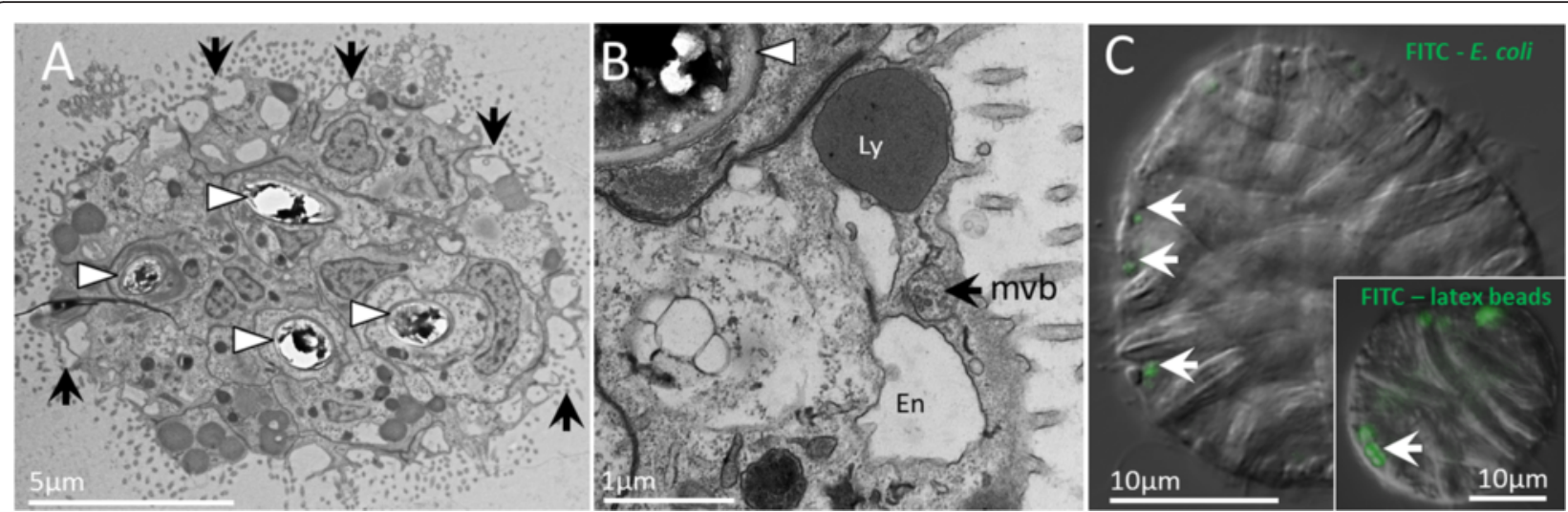

Fig. 6 Phagocytes in nematosomes. a A thin section of a nematosome showing four cnidocytes (white arrowheads) and at least five cells with abundant apical vacuoles (black arrows). b At higher magnification, several organelles are visible: (mvb) multivesicular body, (Ly) lysosome-like vesicle, and (En) endosome-like vesicle. c An optical section through a single nematosome showing sub-surface localization of fluorescently-labeled $E$. coli (white arrows) and latex beads (inset)

To evaluate the quality of our three transcriptomes, we first examined each for the number of transcripts and open reading frames (ORFs) they generated. To minimize the number of spurious ORF predictions, we used the default settings in Transdecoder (http://transdecoder.github.io/) which limits predictions to only those sequences with an ORF $>100$ amino acids (aa) in length. We further examined the number of expressed transcripts by mapping our complete set of reads back to each reference transcriptome using Bowtie2 [54]; aligned reads were then counted with the multiBamCov utility in BedTools [55]. Finally, we examined each transcriptome for completeness with CEGMA [56], which uses BLAST to search a set of 248 conserved eukaryotic genes against each assembled transcriptome. CEGMA scores represent the percent of the 248 conserved genes that were present in each assembly; assemblies with higher CEGMA scores are considered to be more complete.

The full transcriptome comprised 333,942 transcripts but only $9 \%$ of these (29,690 transcripts) encoded ORFs $>100$ aa (Table 1), suggesting the majority of the assembled transcripts are very short. Additionally, each ORF-encoding transcript produced an average of 4.6 unique ORFs. While this resulted in 135,213 total predicted ORFs, the majority of these putative proteins (55\%,74,503 transcripts) were estimated to have no expression (total count $<2$ across tissues). Together, these data suggest that TR333942 does indeed contain a large number of erroneously assembled transcripts, however, this assembly also had the highest CEGMA score, as 242/248 (98 \%) conserved eukaryotic genes from the CEGMA database were present in this transcriptome. Thus, TR333942 is useful for identifying complete transcript sequences but not for assaying differential expression across tissues.
The transcriptome assembled from one trimmed replicate of each tissue (TR118377) produced 118,377 transcripts, $16 \%$ of which $(19,461$ transcripts) encoded long ORFs; this assembly, therefore, resulted in fewer erroneously assembled short transcripts than TR333942. However, those 19,461 transcripts produced 77,877 predicted ORFs, which equates to an average of approximately 4 unique ORFs per transcript. Like TR333942, TR118377 was characterized by a large number of sequences (28,975 transcripts) with no expression and also a very high CEGMA score, making this transcriptome also useful for identifying complete transcript sequences but not expression analyses.

Finally, the reference transcriptome assembled from trimmed/error corrected reads from a single replicate of each tissue (NvecRef32743) produced 32,743 transcripts, most of which (53\%, 17,346 transcripts) encoded long ORFs and produced only 1.6 ORFs per transcript. This resulted in 27,511 total predicted ORFs, $37 \%$ of which (10,198 transcripts) were estimated to have no expression. The completeness of this reduced transcriptome was lower than either TR333942 or TR118377 (83 \% vs 98/ $97 \%$ ) suggesting that error correction limited our ability to assemble full length transcripts. As an additional measure of assembly quality, we explicitly examined mapping concordance (percent of curated reads that mapped back to the NvecRef32743 assembly) following trimming and error correction (Table 2). Over $75 \%$ of the reads from each sample mapped back to our reference assembly and error correction increased this number considerably (average $8.6 \%$ increase in concordance). Given these metrics of assembly quality and our goal of confidently assembling truly novel transcripts we chose to use NvecRef32743 assembled from trimmed/error-corrected reads as our reference transcriptome for differential expression and 
Table 1 Comparison of transcriptome assembly statistics

\begin{tabular}{llll}
\hline & Trimmed only, N=3 reps/tissue & Trimmed only, N=1 rep/tissue & Trim + error correction, $N=1$ rep/tissue \\
\hline Transcriptome name & TR333942 & TR118377 & NvecRef32743 \\
Assembled "transcripts" $^{\text {"a }}$ & 333,942 & 118,377 & 32,743 \\
Transcripts with predicted ORFs $^{\mathrm{b}}$ & $29,690(9 \%)$ & $19,461(16 \%)$ & $17,346(53 \%)$ \\
Predicted ORFs $^{c}$ & $135,213(4.6 \mathrm{X})$ & $77,877(4 \mathrm{X})$ & $27,511(1.6 \mathrm{X})$ \\
ORFs with no expression $^{\mathrm{d}}$ & $74,503(55 \%)$ & $28,975(37 \%)$ & $10,198(37 \%)$ \\
CEGMA score (complete) $^{\text {e }}$ & $242 / 248(98 \%)$ & $241 / 248(97 \%)$ & $207 / 248(83 \%)$ \\
CEGMA score (partial) & $245 / 248(99 \%)$ & $245 / 248(99 \%)$ & $231 / 248(93 \%)$
\end{tabular}

${ }^{a}$ Total number of contigs assembled using Trinity $[49,50] .{ }^{b}$ Open reading frames (ORFs) $\geq 100$ amino acids (aa) in length were predicted using Transdecoder (http://transdecoder.github.io). ${ }^{\mathrm{C}}$ The average number of ORFs predicted from each transcript is listed in parentheses. ${ }^{\mathrm{d}}$ Total number of transcripts with at least one ORF and abundance estimated at $<2$ counts in at least one of the three sampled tissues; counts were assayed using Bedtools [55]. ${ }^{\text {e } C E G M A ~ s c o r e s ~ a r e ~ l i s t e d ~ a s ~ \% ~}$ of the 248 conserved eukaryotic genes that form the CEGMA database [56] that were present in the indicated transcriptome (scores for complete and partial sequences are indicated)

orthology analyses. Our differential expression and qPCR analyses (see below) confirm that NvecRef32742 accurately represents the distribution of transcripts across tissues, supporting our decision to take this conservative approach.

To ensure all assembled transcripts in our reference transcriptome were from $N$. vectensis, we used the program alien_index (https://github.com/josephryan/alien_index) to identify putative foreign/contaminating transcripts. From the assembled transcriptome of 32,743 transcripts, we identified 82 sequences of potential foreign origin and examined each manually using blastn in the published $N$. vectensis genome. Those sequences with alien index scores greater than 0 , i.e., with a high likelihood of foreign origin ( $N=82$ sequences, available in Additional file 7) that also had high quality BLAST hits in the genome ( $>70 \%$ identity and $>200$ bp in length, $N=45$ sequences) were retained for further analysis; all other sequences were considered putative contaminants ( $N=37$ sequences) and were removed (see summary of alien index analysis in Additional file 8). In summary, our cleaned reference transcriptome consisted of $32,706 N$. vectensis transcripts which encoded
17,313 predicted ORFs, 2150 of which lack models in the published JGI database (Table 3; Additional file 8).

Our goals for differential expression and orthology analyses were three-fold: first, we wanted to generate a molecular profile of the nematosomes by describing the types of genes expressed in this tissue with a specific focus on any novel transcripts that might have been missed in previous sequencing projects. Second, we wanted to explicitly test the hypothesis that the nematosome transcriptome comprises a subset of the genes expressed in the mesenteries (from which nematosomes arise) and further, that nematosomes share more genes in common with the mesenteries than with the tentacles. Third, we aimed to evaluate the hypothesis that Nematostella-specific genes are restricted to (or overrepresented in) this novel tissue.

\section{Nematosomes are molecularly distinct from mesenteries and tentacles}

We used principal components analysis to assess variation among replicates and across tissues. While variation was highest among the nematosome samples, all three tissues

Table 2 Error correction increases the mapping concordance for transcriptome NvecRef32743

\begin{tabular}{|c|c|c|c|c|c|}
\hline Sample $^{a}$ & $\begin{array}{l}\text { Raw reads } \\
\text { (paired) }\end{array}$ & $\begin{array}{l}\text { Reads remaining after } \\
\text { trimming }\end{array}$ & $\begin{array}{l}\text { Reads remaining after trimming \& } \\
\text { Error Correctionc }\end{array}$ & $\begin{array}{l}\text { Reads aligned }{ }^{\mathrm{d}}- \\
\text { Trimmed only }\end{array}$ & $\begin{array}{l}\text { Reads aligned - Trimmed \& } \\
\text { Error Corrected }\end{array}$ \\
\hline M3 & $49,390,203$ & $49,377,414$ & $46,888,223$ & $39,768,569(81 \%)$ & $43,249,696$ (92 \%) \\
\hline M4 & $47,477,373$ & $47,466,721$ & $44,704,490$ & $36,255,081$ (76 \%) & 39,339,951 (88 \%) \\
\hline Mes & $26,632,325$ & $26,615,206$ & $25,785,788$ & $24,435,421(92 \%)$ & $24,380,462$ (95 \%) \\
\hline N1 & $47,788,007$ & $47,770,601$ & $44,558,275$ & $36,611,388$ (77 \%) & $39,656,864$ (89 \%) \\
\hline N2 & $53,500,454$ & $53,483,271$ & $50,693,980$ & $42,513,852$ (79 \%) & $45,609,374$ (90 \%) \\
\hline Nem & $20,135,090$ & $20,073,479$ & $19,174,413$ & $17,995,873$ (90 \%) & $17,834,121(93 \%)$ \\
\hline T3 & $47,683,996$ & $47,670,541$ & $44,585,027$ & $27,963,673$ (77 \%) & $39,854,556(89 \%)$ \\
\hline $\mathrm{T} 4$ & $50,314,893$ & $50,300,293$ & $47,474,608$ & $40,154,724(80 \%)$ & 43,557,953 (92\%) \\
\hline Ten & $21,803,901$ & $21,788,366$ & $20,983,324$ & $19,474,441$ (89 \%) & 19,384,395 (92 \%) \\
\hline
\end{tabular}

${ }^{a}$ Samples M3, M4, Mes are from mesenteries; N1, N2, Nem are from nematosomes; T3, T4, Ten are from tentacles. ${ }^{\mathrm{b}}$ Trimming was performed using Trimmomatic [52]. ' Error correction was performed using the ErrorCorrectReads utility from AllPaths-LG [53]. ${ }^{d}$ Alignments were performed using Bowtie2 [54] 
Table 3 Reference transcriptome NvecRef32743 statistics

\begin{tabular}{ll}
\hline Total number of raw reads pooled for de novo & $137,142,632$ \\
assembly & \\
Assembled "transcripts" & 32,743 \\
Transcripts remaining after alien index analysis & 32,706 \\
Transcripts encoding an ORF > 100aa in length & 27,511 \\
Transcripts with non-0 expression and ORF >100aa & 17,313 \\
New transcripts (not predicted from the genome) & 2,150 \\
Mean transcript length in bp ( $N=32,706 / 17,313)$ & $1,058 / 1,646$ \\
N50 in bp ( $N=32,706 / 17,313)$ & $1,919 / 2,282$ \\
GC content $(N=32,706 / 17,313)$ & $44.56 \% / 45.5 \%$ \\
\hline
\end{tabular}

formed distinct clusters along the first two components (Fig. 7a), confirming that variation among tissues exceeds variation among replicates and that the expression profiles of our three tissues are indeed distinct. To examine differences in gene expression across tissues, we performed hierarchical clustering based on fold-change expression differences in variance stabilized count data using DESeq2 [57] (See Additional file 9 for statistical methods). For this study, we consider only those transcripts with $\geq|2|\left(\log _{2}\right)$ fold-change differences between tissues with a false discovery rate of $<0.05$ as differentially expressed. While numerous transcripts had largely ubiquitous expression across tissues, we also identified clusters of transcripts that were highly expressed exclusively in the nematosomes and clusters of transcripts with high expression in either mesenteries or tentacles (Fig. 7b).

To validate our differential expression patterns, we examined the expression of several transcripts found to be upregulated in the nematosomes using qPCR in independent samples $(N=3)$ of each tissue. We randomly selected twenty transcripts from the set of 528 transcripts identified as being upregulated in nematosomes for quantitative PCR analysis. Nineteen of these twenty transcripts exhibited significantly lower expression $(\geq 2$

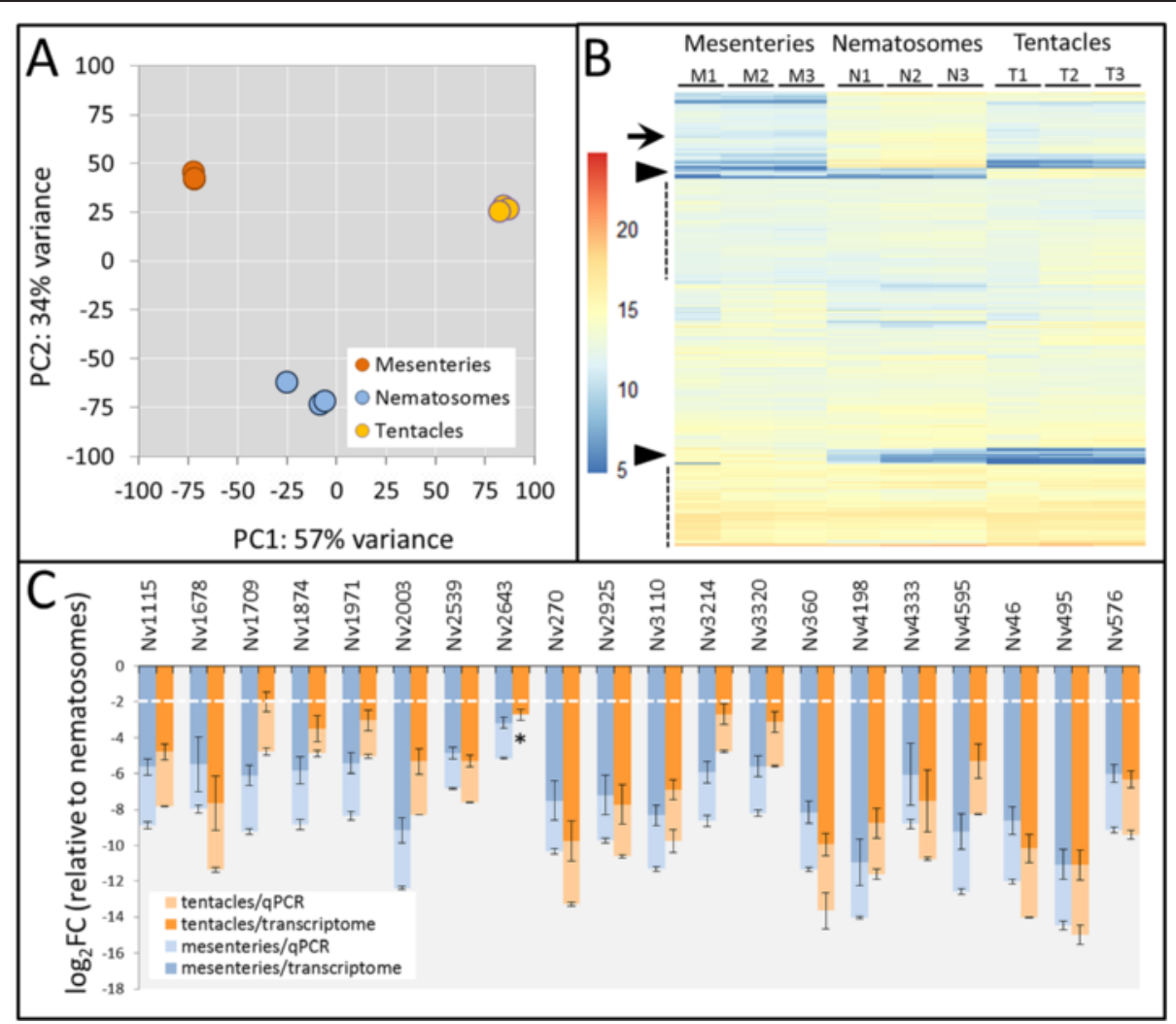

Fig. 7 Expression analyses in three N. vectensis tissues. a Principal components plot indicating variation within and among tissue types. PC1 describes $57 \%$ of the variation among tissues and PC2 describes $34 \%$ in the top 1000 expressed transcripts. b Heatmap indicating gene expression (variance stabilized counts) of the top 1000 expressed transcripts. Transcripts are clustered using the Ward's D method (see Additional file 9); warmer colors indicate higher expression. Dotted lines indicate clusters with ubiquitous expression across tissues, the arrow indicates a cluster with high expression in nematosomes only, and arrowheads indicate clusters with high expression in only mesenteries or tentacles. c Nineteen out of twenty transcripts identified by RNA-Seq as being significantly upregulated in nematosomes relative to both other tissues were also found to have significantly lower expression in the mesenteries and tentacles, relative to nematosomes, using qPCR. Expression of Nv2643 in the tentacles (indicated by $*$ ) is not significantly different from 0 . Dotted lines indicate $2\left(\log _{2}\right)$-fold change in expression between nematosomes and the other tissues. Mean \pm SD 
$\log _{2}$ fold lower) in both the mesenteries and tentacles relative to the nematosomes (Fig. 7c). Together, these data suggest that our approach was effective at identifying differentially expressed transcripts across tissues.

\section{Lineage-specific genes are overrepresented in nematosomes (and all tissues)}

To characterize the types of transcripts upregulated in each tissue, we performed orthology analysis $[58,59]$ and assayed the distribution of transcripts of different taxonomic origins in each tissue. Specifically, we examined the distribution of Nematostella-specific genes, genes found only in representatives of the Edwardsiidae, genes found only among anthozoans, those found only among cnidarians, and conserved genes shared across metazoans (see Methods for a description of the specific taxa included in each orthology group). In the complete dataset (17,313 ORF-encoding transcripts), the largest proportion of transcripts (60\%, 10,413 transcripts) grouped with orthologs from across Metazoa, whereas Nematostella-specific transcripts comprised only $16 \%$ of the dataset (2792 transcripts) (Fig. 8a). A previous study estimated the number of Nematostella-specific genes to comprise approximately $11 \%$ of the genome [7], suggesting our efforts to assemble a reference transcriptome de novo was an effective method for identifying additional predicted genes.
Conserved/metazoan transcripts represented a smaller proportion of the upregulated genes in all tissues (Fig. 8b-d) than in the whole dataset (Fig. 8a). Of the 528 transcripts found to be upregulated specifically in nematosomes ( $\geq 2 \log _{2}$ fold higher expression in nematosomes relative to both other tissues), only $32 \%$ (170 transcripts) grouped with orthologs from diverse metazoans. Nematostella-specific transcripts comprised $26 \%$ of the upregulated genes from nematosomes (135 transcripts) and fully $68 \%$ of the transcripts in the upregulated genes in this dataset were cnidarian-specific (Fig. 8b). This pattern was conserved across tissues, with Nematostella-specific genes comprising $26 \%$ of the upregulated genes from the mesenteries (Fig. 8c) and $30 \%$ of the tentacle dataset (Fig. 8d), and the majority of the transcripts in both mesenteries and tentacles $(63 \%$ and $74 \%$, respectively) also grouping only with other cnidarian sequences. We further examined the subset of transcripts that were expressed uniquely in each tissue (Fig. 8e-g) to test the hypothesis that Nematostellaspecific genes are expressed uniquely in a Nematostella-specific tissue. Of the 528 upregulated transcripts in the nematosomes (Fig. 8b), 52 were found to be expressed uniquely in this tissue (Fig. 8e). The majority of these uniquely expressed genes (65\%, 34 transcripts) were indeed Nematostella-specific but, surprisingly, 90 \% (47 transcripts) of them grouped only with other cnidarian

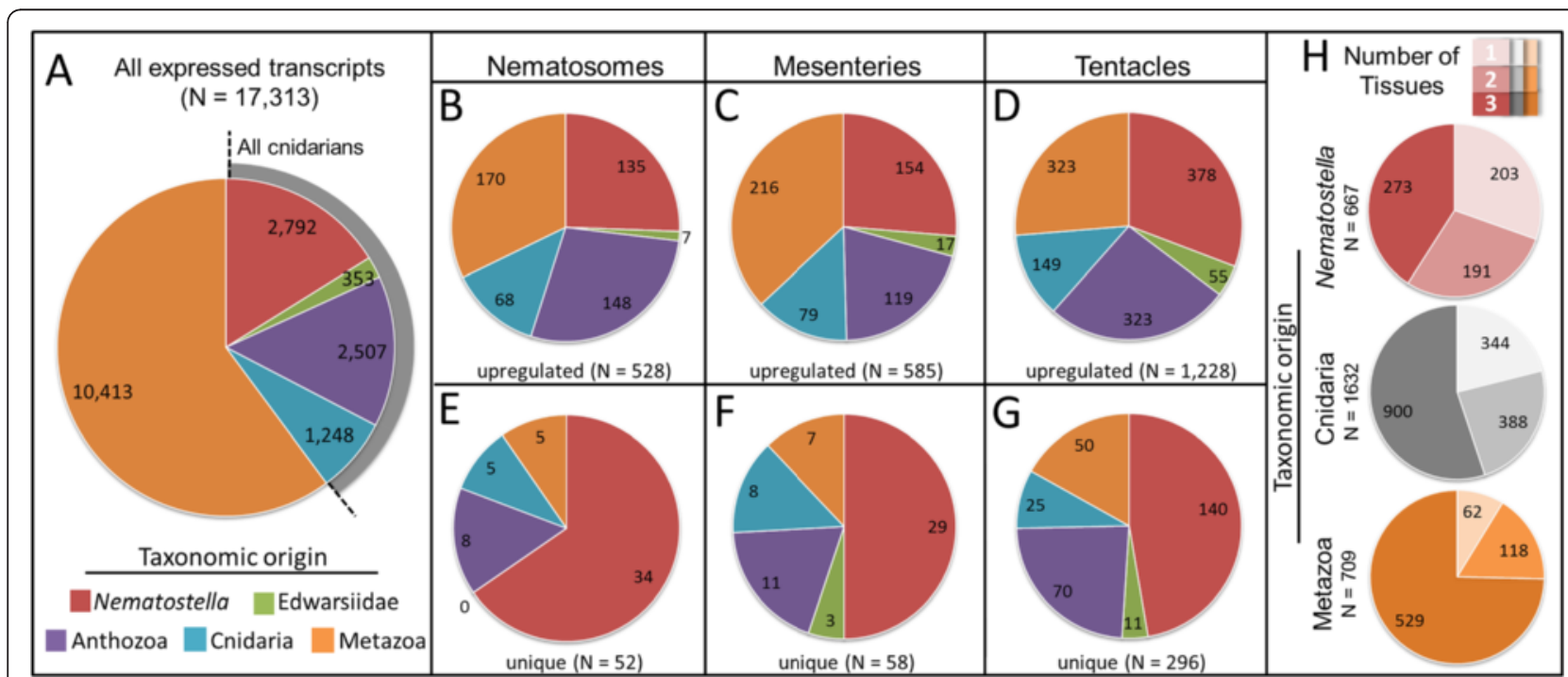

Fig. 8 Analysis of taxon-restricted gene expression. a Of 17,313 expressed transcripts identified in this study, approximately $16 \%(N=2792)$ are Nematostella-specific, $2 \%(353)$ are Edwardsiidae-specific, $14 \%(N=2507)$ are anthozoan-specific, and $7 \%(N=1248)$ are cnidarian-specific. The largest class of transcripts sequenced in this study (60\%) were found in at least one other group of metazoans $(N=10,413)$. As compared with the complete set of expressed transcripts, (b-d) differentially expressed transcripts were characterized by a higher proportion of Nematostella-specific sequences: $26 \%$ in nematosomes and mesenteries ( $N=135 / 528$ and $N=154 / 585$, respectively) and $31 \%$ in tentacles $(N=378 / 1228)$. Shared/metazoan sequences comprised less than half of the differentially expressed transcripts in each of the three tissues. e-g Nematostella-specific sequences make up an even larger proportion of the uniquely expressed transcripts in each tissue: $65 \%(N=34 / 52)$ in the nematosomes, $50 \%(N=29 / 58)$ in the mesenteries, and $47 \%(N=140 / 296)$ in the tentacles. $\mathbf{h}$ Among the differentially expressed transcripts (illustrated in $\mathbf{b}$-d), Nematostella-specific sequences were more likely to be expressed in only a single tissue $(35 \%, N=203 / 667)$ than were pan-cnidarian sequences (illustrated in grey in panel a; $21 \%, N=344 / 1632)$ or shared/metazoan sequences ( $9 \%, N=62 / 709$ ) 
sequences. Genes expressed uniquely in both mesenteries and tentacles showed a similar pattern (Fig. 8f, g): in the mesenteries $50 \%$ were Nematostella-specific and $88 \%$ cnidarian-specific, and in the tentacles $47 \%$ were Nematostella-specific and $83 \%$ were cnidarian-specific. Thus, Nematostella-specific transcripts comprise a larger proportion of the uniquely expressed transcripts in nematosomes $(65 \%)$ than in both other tissues $(50 \%$ in mesenteries, $47 \%$ in tentacles), but the largest number of Nematostella-specific transcripts was expressed uniquely in the tentacles (140 transcripts). Broadly, cnidarianspecific sequences dominate the set of differentially expressed transcripts in all tissues.

Finally, we ignored tissue type and examined the proportion of transcripts from each orthology group expressed in one or more tissues (Fig. 8h). Thirty percent of the differentially expressed Nematostella-specific transcripts (203/ 667 transcripts) were expressed only in a single tissue. By contrast, only $21 \%$ of the cumulative group of cnidarianspecific transcripts (344/1632 transcripts) were expressed uniquely, as were only $9 \%$ (62/709) of the conserved/metazoan transcripts (Fig. 8h). Together, these patterns provide further evidence that lineage-specific transcripts tend to be tissue-restricted whereas conserved/metazoan transcripts tend to be expressed across multiple tissues.

\section{Functional annotation supports a role in immunity}

To characterize the suite of differentially expressed genes from each tissue, we used gene ontology (GO) functional annotation implemented in $\mathrm{R}$ [60] (see Additional file 10 for full GO reports.). First, we examined the functional annotation of the ten transcripts with the highest expression in each tissue (Table 4). While nematosomes are characterized by transcripts with extracellular/ immune function (e.g., golgi autoantigen, hemicentin, thrombospondin), the highest-expressed transcripts in mesenteries were almost exclusively digestive enzymes (e.g., trypsin, chitinase). Intriguingly, highly expressed transcripts from tentacles tended to be unannotated sequences although two sequences appear to be involved in vertebrate immunity: hemicentin and rhamnose binding lectin. These results are consistent with a role for the nematosomes in the immune biology of $N$. vectensis and also suggest, surprisingly, that the functional identity of the nematosomes may be more similar to that of the tentacles than to the mesenteries.

To further explore these hypotheses, we identified the top ten GO terms from nematosomes (for each GO category: molecular function, biological process, and cellular component) and compared the number of transcripts that mapped to each term across tissues (Fig. 9a). Two GO terms associated with potassium ion activity (MF: voltage-gated $\mathrm{K}^{+}$channel activity, BP: $\mathrm{K}^{+}$ion transmembrane transport) were found to be over-represented in nematosomes relative to both other tissues (Fig. 9a, white arrowheads), confirming the unique identity of this tissue and suggesting potentially novel cell physiology or cell signaling processes in this tissue. These analyses also revealed several GO terms that were common to nematosomes and tentacles but were absent from the mesenteries (Fig. 9a, black arrowheads), including terms associated with arachidonic acid metabolism, known for its important roles in cell signaling and inflammation $[61,62]$. Overall, nematosomes were found to have $141 \mathrm{GO}$ terms in common with mesenteries and 341 with tentacles (Fig. 9b, Additional file 10).

Finally, we assayed the set of upregulated transcripts $(N=528)$ from the nematosomes explicitly for genes associated with immunity using a reciprocal BLAST approach and a previously published database of stress genes from the $N$. vectensis genome [63]. This analysis revealed that over $20 \%$ of the transcripts found to be upregulated in nematosomes (22\%, 116/528 transcripts) had reciprocal best BLAST hits with genes commonly associated with wound, pathogen, or chemical stressors (Table 5, Additional file 8). By comparison, only $17 \%$ (101/585 transcripts) of the upregulated transcripts from the mesenteries had significant hits in this stress database and this value dropped to $13 \%(161 / 1228$ transcripts) for the tentacles. The stress categories "wound" and "pathogen" were associated with the most transcripts from each of the three tissues, but the distribution of transcripts annotated to these categories varied by tissue. Whereas transcripts associated with pathogen stress comprised the majority of the transcripts from nematosomes $(75 \%)$, less than $70 \%$ of the transcripts from tentacles were associated with pathogen stress and only $56 \%$ of the transcripts from mesenteries were associated with this stress category. Wound-associated transcripts comprised $33 \%$ and $37 \%$ of the transcripts from nematosomes and tentacles, respectively, and nearly $50 \%$ of the transcripts from mesenteries. In summary, several pieces of data point to a novel immune capacity for the nematosomes.

\section{Discussion}

Evaluating the cell biology and function of nematosomes Our data provide support for several previous hypotheses about the function of nematosomes, including their role in the prey capture and defense, and a novel potential role in the immune system of $N$. vectensis. In support of Hand [32], we confirm that the cnidocytes in nematosomes are capable of subduing prey (Fig. 3, Additional file 4), and that this function results from the firing of their abundant cnidocytes. However, like Williams [33], we find no evidence of gland cells in nematosomes; thus, our results are in conflict with the recent suggestion that the venom responsible for subduing prey derives from ectodermal 
Table 4 Pfam/Conserved domain analysis of the top expressed transcripts from each tissue

\begin{tabular}{|c|c|c|c|c|c|}
\hline Tissue & TrID & GrID & $J G I I D$ & $\mathrm{Pfam} / \mathrm{CD}(\mathrm{s})^{\text {a }}$ & Description \\
\hline \multirow[t]{10}{*}{ nematosomes } & Nv270 & M & 200843 & Myosin_tail_1, Cast, TPR_MLP1_2, Mplasa_alph_rch & golgi autoantigen $\mathrm{B} 1$ \\
\hline & Nv46 & $\mathrm{N}$ & 239786 & cadherin & uromodulin \\
\hline & Nv1832 & C & N/A & Calponin homology $(\mathrm{CH})$ domain, Spectrin repeat, SMC_prok_A, PRK02224 & utrophin \\
\hline & Nv495 & C & 246793 & $\begin{array}{l}\text { reprolysin, pep_M12B_propeptide, ADAM_spacer1 super family, TSP_1, FN3, ZnMc } \\
\text { super family, ADAM_CR super family }\end{array}$ & ADAMTS \\
\hline & Nv791 & C & 27059 & $\begin{array}{l}\text { ZnMc_adamalysin_II_like, reprolysin, pep_M12B_propeptide, F5_F8 type C, } \\
\text { disintegrin, ADAM_CysRich, TSP_1, FReD super family, EB }\end{array}$ & $\begin{array}{l}\text { hemicentin/ } \\
\text { thrombospondin }\end{array}$ \\
\hline & Nv126 & C & 242847 & N/A & uncharacterized \\
\hline & Nv354 & M & 119733 & Homeobox KN domain, PRK12323 Sox_C_TAD super family & $\begin{array}{l}\text { Meis2 transcription } \\
\text { factor }\end{array}$ \\
\hline & Nv111 & $\mathrm{N}$ & 200034 & $\mathrm{~N} / \mathrm{A}$ & uromodulin \\
\hline & Nv1971 & A & 96874 & FN3, TLD, MAM, F5_F8_typeC, Ion_trans_2 & $\begin{array}{l}\text { sidekick-2 } \\
\text { (immunoglobulin) }\end{array}$ \\
\hline & Nv1874 & M & 21107 & $\begin{array}{l}\text { PKD channel, REJ, PLAT_polycystin, PKD, GPS, F5_F8_typeC, WSC, DUF4271 super } \\
\text { family }\end{array}$ & cation channel \\
\hline \multirow[t]{10}{*}{ mesenteries } & Nv2 & M & 39271 & CstA, PLA2C & Phospholipase A2 \\
\hline & Nv151 & M & 205229 & PHA03307, DUF1943, WWD, Vitellogenin_N & vitellogenin precursor \\
\hline & Nv169 & M & 180912 & CBM_14 & chitin binding protein \\
\hline & Nv224 & M & 168629 & $\begin{array}{l}\text { GH18_chitolectin_chitotriosidase, Glyco_18, Retinal, PHA03307, DUF2237 super } \\
\text { family }\end{array}$ & chitinase \\
\hline & Nv274 & M & 109239 & ND2 super family, Tryp_SPc, SR & chymotrypsin \\
\hline & Nv174 & M & 105779 & Atrophin-1, Tryp_SPc, ShKT & chymotrypsin \\
\hline & Nv158 & $\mathrm{N}$ & 246653 & LDLa, UPF0104 super family, Tymo_45kd_70kd & $\begin{array}{l}\text { uncharacterized } \\
\text { membrane protein }\end{array}$ \\
\hline & Nv215 & M & 15302 & ZnMc_MMP, PG_binding_1, ShKT, Peptidase_M10 & $\begin{array}{l}\text { matrix } \\
\text { metalloprotease }\end{array}$ \\
\hline & Nv9181 & M & 246069 & Mito_carr, PTZ00169 & $\begin{array}{l}\text { ADP,ATP carrier } \\
\text { protein }\end{array}$ \\
\hline & Nv896 & C & 98917 & $\begin{array}{l}\text { ZnMc_astascin like, ShK toxin domain, astacin (Peptidase family M12A), prolyl 4- } \\
\text { hydroxylase }\end{array}$ & $\begin{array}{l}\text { matrix } \\
\text { metalloprotease }\end{array}$ \\
\hline \multirow[t]{10}{*}{ tentacles } & Nv302 & C & N/A & DnaJ_zf, GPS, TIG, Glyco_hydro_17 super family, REJ & egg jelly receptor \\
\hline & Nv520 & M & 120496 & PTKc, FN3, SEA, Ig_2, Pkinase_Tyr, IGc2 & FGF receptor c \\
\hline & Nv146 & M & 90289 & KBL_like, Preseq_ALAS super family, BioF & aminotransferase \\
\hline & Nv886 & M & 80526 & Ig, Ig super family, Ig2_FGFR_like, Ig super family, I-set, IG_like, IG, IGc2 & $\begin{array}{l}\text { Immunoglobulin like } \\
\text { activity }\end{array}$ \\
\hline & Nv94 & $\mathrm{C}$ & 114661 & SOUL & heme-binding protein \\
\hline & Nv139 & $\mathrm{N}$ & 122916 & Gal_lectin, FYDLN_acid super family & $\begin{array}{l}\text { rhamnose binding } \\
\text { lectin }\end{array}$ \\
\hline & Nv325 & M & 202708 & YtcJ_like & aminohydrolase \\
\hline & Nv219 & M & 243257 & Gln-synt_C, Gln-synt_N, PLN02284 & glutamine synthetase \\
\hline & Nv326 & $\mathrm{N}$ & 208719 & TSP1, WSC super family & hemicentin \\
\hline & Nv162 & $\mathrm{N}$ & 202441 & DUF2457 & uncharacterized \\
\hline
\end{tabular}

\footnotetext{
a Protein family (Pfam) domains were identified from the EMBL-EBI database (http://pfam.xfam.org/) and conserved domains (CD) were identified from NCBI's conserved domain database (http://www.ncbi.nlm.nih.gov/Structure/cdd/wrpsb.cgi). TrID internal transcript ID from NvecRef32743 de novo assembly, GrID orthology group, N Nematostella-specific, A anthozoan-specific, C cnidarian-specific, $M$ shared/metazoan, JGI ID protein ID from the JGI database (http://genome.jgi-psf.org/Nemve1/Nemve1.home.html)
}

gland cells adjacent to the cnidocytes in $N$. vectensis, rather than the cnidocytes themselves [64]. As noted in the early descriptions of nematosomes (summarized in: [32]), spirocytes are absent from this tissue, so it is possible, though speculative, that ectodermal gland cells work in tandem with spirocytes, which is why both are abundant in the tentacles.

Cnidocytes were once thought to behave as "independent effectors" responding autonomously to stimulus [65] 


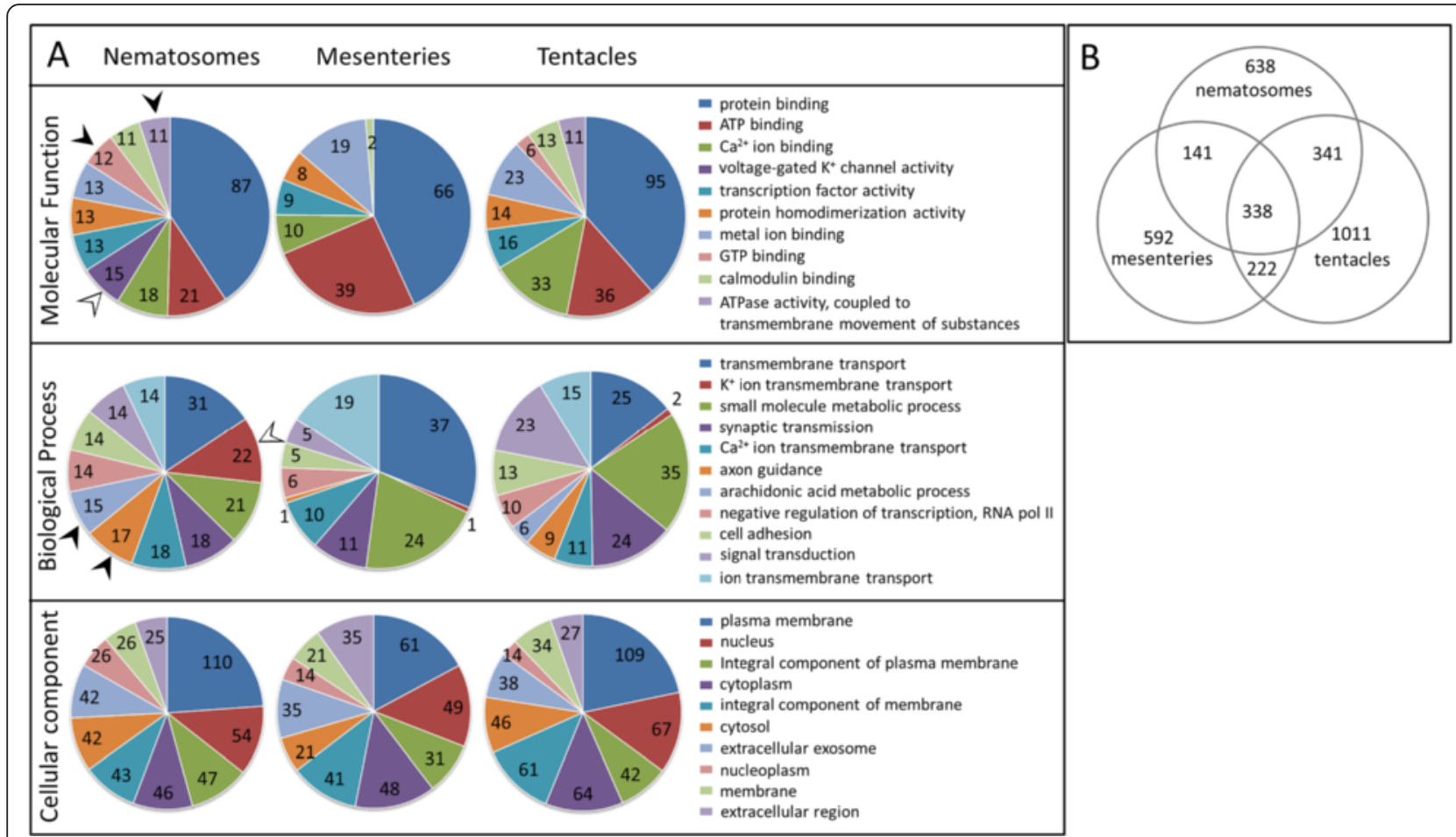

Fig. 9 Gene ontology (GO) analyses of RNA-Seq data. a The top 10 GO terms from nematosomes for each GO category and the number of transcripts that map to them from other tissues. White arrowheads indicate $\mathrm{GO}$ terms unique (or nearly so) to nematosomes and black arrowheads indicate $\mathrm{GO}$ terms shared only by nematosomes and tentacles. $\mathbf{b}$ Venn diagram showing the number of $\mathrm{GO}$ terms shared among tissues

but subsequent studies have revealed that this behavior may be modulated by chemical and mechanical stimuli [66] and by neuronal/synaptic input $[67,68]$. The ultrastructural data presented in Fig. 5 suggest that nematosomes lack the sensory cell complexes necessary for mechanosensitization and neuronal modulation of cnidocyte discharge [69]; however, certain types of cnidocyte/ support cell complexes are known to fire either as a result of physical contact alone or in response to combined physical and chemical stimuli [70]. Currently, we cannot discriminate between these two possibilities in nematosomes but future studies aimed at localizing chemoreceptors in the putative nematosome support cells will provide important insight on this topic. Furthermore, the absence of neurons from nematosomes may suggest that this tissue has evolved a means to limit the firing response of their cnidocytes to only physical stimuli, perhaps as a means to maximize firing success while nematosomes are "tumbling" around in the gastrovascular cavity.

Beyond the ability to subdue prey, we propose that nematosomes may also play a role in defense of the spawned egg masses. Unlike broadcast spawning species of sea anemone, $N$. vectensis produces egg masses bound by a jelly matrix $[26,34,35]$. Although the lifespan of nematosomes is reported by some to be $<21 \mathrm{~h}$ at room temperature [25], we routinely observed living nematosomes embedded in the egg jelly as planulae larvae begin to swim and emerge from the egg mass $(>48 \mathrm{~h}$ after fertilization at $25{ }^{\circ} \mathrm{C}$ ) (see Additional file 11 and [35]). Considering this long lifespan and the proximity to the

Table 5 Abundance of candidate stress genes across tissues

\begin{tabular}{llll}
\hline & Nematosomes & Mesenteries & Tentacles \\
\hline Transcripts assayed $^{a}$ & 528 & 585 & 1228 \\
Transcripts with reciprocal best hits in stress database ${ }^{b}$ & $116(22 \%)$ & $101(17 \%)$ & $161(13 \%)$ \\
Wound $(N=741)$ & $38(33 \%)^{c}$ & $46(46 \%)$ & $59(37 \%)$ \\
Pathogen $(N=1984)$ & $87(75 \%)$ & $57(56 \%)$ & $109(68 \%)$ \\
Chemical $(N=187)$ & $6(2 \%)$ & $9(9 \%)$ & $10(6 \%)$ \\
\hline
\end{tabular}

${ }^{\mathrm{a}}$ The complete set of upregulated transcripts for each tissue. ${ }^{\mathrm{b}}$ Database of putative stress-related transcripts identified by Reitzel et al [63] for the categories: Wound, Pathogen, and Chemical. For a list of genes in each tissue/class see Additional file 8. 'Values represent the number (percent) of the transcripts with reciprocal best hits in the stress database that were associated with the indicated stressor. The sum of these values exceeds the number of transcripts with reciprocal best hits because some transcripts had equally high BLAST hits in two different stress categories 
developing embryos in the egg mass, nematosomes could reasonably play a role in defense of the spawned egg masses. Anecdotal observations of live killifish behavior also suggest that these potential predators may be deterred by cnidocyte discharge from the abundant nematosomes embedded in the jelly matrix (Adam Reitzel, pers. comm.). Together, these data suggest that nematosomes are an important component of the ecology of $N$. vectensis.

Our data also suggest that nematosomes may play an important part in the immune system of $N$. vectensis. Inflammation in cnidarians has been described as a global phagocytic response ([71], as translated by [72]). In support of this claim, the few previous studies of anthozoan immune function have demonstrated a dramatic increase in the number of mobile phagocytic cells following injury $[72,73]$. Immune function has not been functionally characterized in $N$. vectensis but nematosomes might be an important reservoir for the phagocytes that comprise the putative inflammatory response in this species. Figure 6 demonstrates the propensity of cells in the nematosomes to engulf particles of various types (E. coli and latex beads), confirming the presence of phagocytes in this tissue. Furthermore, two of the top-expressed transcripts in the nematosomes share homology with uromodulin (Nv46 and Nv111), a glycoprotein known to be involved in protein-protein interactions associated with the inflammatory response of mammals $[74,75]$ and previously shown to be upregulated during wound-healing in $N$. vectensis [76]. Although the behavior of nematosomes during wound-healing/regeneration has not been studied, the presence of phagocytes suggests this tissue may play a role in clearing damaged tissue from a wound site. In this regard, it would be very interesting to determine if nematosomes aggregate at the site of a wound in $N$. vectensis.

We show that nematosome size is associated with its cellular composition (Fig. 4d); while cnidocytes make up half of the cells present in small nematosomes, they comprise only $\sim 30 \%$ of the cells in large nematosomes. We have also shown that nematosomes do not typically contain proliferative cells and likely do not undergo growth after they leave the mesentery epithelium, meaning that nematosome size is determined during the development and budding processes. Given that larger nematosomes have more non-cnidocyte cells than smaller nematosomes, and that at least some of these cells are phagocytes, we suggest that both nematosome size and budding rate may correlate positively with the animal's perceived pathogen load. In this light, it would be interesting to raise $N$. vectensis polyps in the presence of antibiotics to determine if nematosome number decreases in this pathogen-free environment. We could further hypothesize that pathogen-related genes might be upregulated in nematosomes from polyps exposed to higher pathogen loads.

\section{Toward a molecular identity of nematosomes}

The data presented herein represent the first tissuespecific transcriptome data from $N$. vectensis, an important model for cell biology and development, and the first molecular description of the nematosomes. Using these molecular resources, we evaluated two hypotheses about evolutionary innovation in $N$. vectensis. First, because nematosomes bud from the mesenteries and contain the same two types of cnidocytes found in the mesenteries (basitrichs and microbasic p-mastigophores), we hypothesized that the transcriptional profile of the nematosomes would constitute a subset of the transcripts expressed in the mesenteries. Differential expression, qPCR, and GO analyses independently verify the unique gene expression profile of the nematosomes (Figs. 7 and 8 ) and reinforce their putative role in immunity (Fig. 9, Tables 4 and 5) but also suggest that nematosomes may have more cell types or cell functions in common with tentacles than with mesenteries. These similarities in gene expression between nematosomes and tentacles may reflect the relative abundance of cnidocytes in these tissues, suggesting this dataset will be important for identifying novel markers of cnidocyte and support cell identity. Additionally, because mesenteries give rise to new nematosomes, the genes responsible for specifying this novel tissue at the onset of its development must be among the mesentery-specific dataset. Future studies aimed at identifying these nematosome developmental genes would be extremely valuable in understanding the evolutionary and developmental origin of this novel tissue. Thus, using comparative transcriptomics, we reject the hypothesis that nematosomes are merely sloughed portions of the mesenteries and suggest that nematosomes constitute a novel tissue with a unique identity and have characteristics consistent with cell functions that have not yet been characterized in $N$. vectensis.

The second hypothesis that we evaluated with these comparative transcriptome data was motivated by the assertion that lineage-specific genes are expected to be expressed in higher frequency in lineage-specific tissues $[5,77]$. Given that nematosomes are unique to the genus Nematostella, we expected Nematostella-specific genes to be overrepresented in the nematosomes, relative to the two other tissues, one of which is specific to anthozoans (mesenteries) and the other is specific to cnidarians (tentacles). We found that Nematostella-specific genes comprised a larger proportion of the transcripts expressed uniquely in nematosomes than in either mesenteries or tentacles, but the largest number of Nematostella-specific transcripts was expressed uniquely in the tentacles. These results can be interpreted in several 
ways: first, the broader expression of conserved genes may simply reflect that a large proportion of the conserved genes we sequenced play a role in conserved functions that are common to all cells (i.e., "housekeeping" functions). Second, this pattern could reflect that conserved genes are expressed in cell types common to all three tissues, yet this hypothesis is harder to support considering that the only cell types known to populate all three tissues are cnidocytes. A third possibility is that this pattern reflects a greater level of pleiotropy in older genes. Indeed, novel genes are thought to arise rapidly [5] and have been shown by us and others to be uniquely expressed in adult tissues (e.g., [12, 16, 77]); whether these novel genes all acquire functions important for the phenotype of the tissue, however, is an unanswered question. Thus, despite the abundance of novel sequences produced, the percent of novel genes that actually take on a critical role in the biology of any given tissue may be relatively modest.

Although uniquely expressed transcripts were dominated by Nematostella-specific genes in all tissues, conserved/metazoan genes were among the top expressed transcripts from all three tissues (3/10 in nematosomes, $8 / 10$ in mesenteries, and $5 / 10$ in tentacles; Table 4). These data are interesting in the context of two recent studies of the role of novel genes in the evolution of novel hymenopteran social behaviors [12, 77]. Like us, Jasper et al [12] found that the proportion of lineagespecific genes in honey bees varies only little across tissue types but they suggest that the level of expression of these novel genes is responsible for driving the novel tissue identity. They show that the greatest proportion of gene expression in the venom/stinger and hypopharyngeal glands (both tissues with novel functions in the honey bee) is attributable to transcription of novel genes, but the patterns they describe appear to be driven by the exorbitantly high expression of a single gene in each tissue. In our study, the nematosomes (the novel tissue) were characterized both by a larger proportion of Nematostella-specific (novel) genes and a larger proportion of the top expressed genes were also found to be lineage-specific (pancnidarian).

\section{Are novel genes important?}

It is well-established that both changes in gene regulation $[3,78]$ and changes in the coding sequences of conserved genes [4] drive morphological innovation but this does not preclude novel genes from also playing a critical role in this process [5]. In fact, there are several examples of novel proteins that are essential for defining the identity of the adult cell or tissue types (e.g., venom components [12], antimicrobial peptides [79], and structural proteins like minicollagen [80]). Interestingly, these novel products are largely secreted proteins that appear to have been incorporated into the terminus of an existing gene regulatory network. From an evolutionary perspective, the simplest way to link a novel protein into an existing regulatory network involves the smallest number of connections between the novel gene and the other components of the network [81] and this network connectivity issue may explain why duplicated genes at the ends of pathways may be maintained at a higher rate than duplicated transcription factors [13, 37]. In the context of this study, Nematostella-specific transcripts expressed uniquely in the nematosomes that encode secreted or structural peptides may well have a critical role in dictating the function or phenotype of this novel tissue.

\section{Conclusions}

Cnidarian genome projects have revealed surprising levels of gene conservation between cnidarians and bilaterians; despite this, cnidarians have several cell and tissue types that are not found in other taxa. This study confirms that a novel tissue (nematosomes) plays an important role in the biology of $N$. vectensis and reveals, for the first time, that this novel tissue has a unique gene expression profile that differentiates it from its tissue of origin. Considering there are only 3-4 cell types present in this tissue, this unique molecular profile of nematosomes must be associated with the identity of only few cell types. Nematosomes, therefore, provide a valuable opportunity to evaluate the relationship between the expression of novel genes and the evolution of novel cell and tissue functions. Rather than being intriguing curiosities, novelties provide a unique opportunity to evaluate the mechanisms of evolution that result in the origin of new structures and have ultimately contributed to the morphological and genomic diversity exhibited in the animal kingdom.

\section{Methods \\ Cnidocyte discharge assay}

For discharge assays, live nematosomes were isolated from recently spawned egg masses using a modified protocol for isolating eggs from the egg jelly [82]. Egg jelly was dissolved by gentle shaking in $4 \%$ cysteine (in $1 / 3 \mathrm{X}$ FSW) for $20 \mathrm{~min}$ at $25^{\circ} \mathrm{C}$ in a $15 \mathrm{ml}$ conical vial. Eggs were allowed to settle to the bottom of the conical vial and the supernatant (containing the nematosomes) was removed immediately and filtered first through a $70 \mu \mathrm{m}$ nylon mesh strainer (BD Falcon 352350) to remove debris and then through a $10 \mu \mathrm{m}$ nylon mesh filter (Millipore NY1002500) to collect nematosomes. Isolated nematosomes were washed in 3-5 changes of $1 / 3 \mathrm{X}$ FSW before use. To determine if the cnidocytes in nematosomes are capable of firing, live nematosomes were isolated and then concentrated in a small glass dish. Recently hatched brine shrimp (A. salina) were then introduced 
into this dish and their behavior was digitally recorded with a Canon digital camera mounted on Zeiss Discovery dissecting microscope. Higher magnification images were produced using either a Zeiss M2 compound microscope or a Zeiss LSM 710 confocal microscope.

\section{Tissue labeling and cell counts}

For whole mount tissue analyses (polyps) and analysis of nematosomes isolated from spawned egg masses, tissues were immobilized in $7.14 \% \mathrm{MgCl}_{2}$ for $15 \mathrm{~min}$ at $25{ }^{\circ} \mathrm{C}$, fixed briefly $(\sim 1.5 \mathrm{~min})$ at $25{ }^{\circ} \mathrm{C}$ in $4 \%$ paraformaldehyde with $0.2 \%$ gluteraldehyde in phosphate buffered saline with $0.1 \%$ Tween-20 (PTw) and then fixed for $1 \mathrm{~h}$ at $4{ }^{\circ} \mathrm{C}$ in $4 \%$ paraformaldehyde in PTw. Fixative was removed from tissue with three washes in PTw and tissues were stored at $4{ }^{\circ} \mathrm{C}$ for up to five days before processing. To determine the total number of cells present in nematosomes of various size, we labeled nuclei with a 30-min incubation in $1 \mu \mathrm{M}$ DAPI at $25^{\circ} \mathrm{C}$. Tissues were then washed in PTw and mounted in $80 \%$ glycerol (in PBS) on Rain- $\mathrm{X}^{\circ}$-coated glass slides for imaging. To identify mature cnidocytes, we added a chelating agent $(10 \mathrm{mM}$ EDTA) to the fixative described above and labeled mature cnidocytes by incubation in $143 \mu \mathrm{M}$ DAPI (in PTw) for $30 \mathrm{~min}$ at $25{ }^{\circ} \mathrm{C}$, following the protocol of Szczepaneck et al [83]. For cell counts, confocal z-stacks were rendered into 3D images and digitally counted using Imaris software (Bitplane, Switzerland). Nuclei and cnidocytes were counted separately in different samples of nematosomes and diameter was measured digitally in each nematosome using the Zeiss LSM Image Browser software. To determine if the relationship between total cell number (indicated by nuclear counts) and nematosome size differed from that of cnidocytes over the range of nematosomes investigated, we performed Analysis of covariance (ANCOVA) using the R statistical computing environment [84].

To assess cell proliferation, tissues were incubated for $30 \mathrm{~min}$ at $25{ }^{\circ} \mathrm{C}$ in $100 \mu \mathrm{M} \mathrm{EdU} \mathrm{(in} 1 / 3 \mathrm{X}$ FSW) and visualization was accomplished following the manufacturer's protocol (Invitrogen C10340). To visualize developing cnidocytes, fixed/washed nematosomes and early planula stage embryos $\left(72 \mathrm{~h}\right.$ post fertilization at $16{ }^{\circ} \mathrm{C}$ ) were incubated overnight at $4{ }^{\circ} \mathrm{C}$ with anti-minicollagen primary antibodies [38] diluted in PBS with $0.1 \%$ TritonX (PTx) and $0.1 \%$ bovine serum albumin to the following concentrations: mcol1 (1/300), mcol3 $(1 / 500)$, mcol4 (1/1000). Primary antibody was removed with 3-5 washes in PTx and tissues were then incubated for one hour at $25{ }^{\circ} \mathrm{C}$ in goat-anti-guinea pig (mcol1 and mcol3) or goat-anti-rabbit (mcol4) secondary antibodies (Invitrogen A21450, A21245) at $1 / 250$ in PTx. Unbound secondary antibody was removed with 3 washes (15 mins each) in PTx and tissues were mounted in glycerol. Similar methods were used to visualize the tubulin component of the cilia in nematosomes. Nematosomes were incubated overnight in antiacetylated tubulin primary antibody (Sigma T6743) diluted 1/500 in PTx and with a goat-anti-mouse secondary antibody (Invitrogen A11001) diluted 1/250 in PTx. These tissues were counter-labeled with a 30 -min incubation at $25{ }^{\circ} \mathrm{C}$ in a combination of $1 \mu \mathrm{M}$ DAPI (in PTw) and phalloidin (Invitrogen A12380) diluted $1 / 200$ in PTx to visualize nuclei and F-actin, respectively. Ciliary length in antibody-stained nematosomes was measured digitally using the Zeiss LSM Image Browser software and lengths were compared statistically using analysis of variance (ANOVA) in $\mathrm{R}$ [84]. To investigate the phagocytotic potential of the nematosomes, live isolated nematosomes were incubated overnight $(\sim 18 \mathrm{~h})$ at room temperature with fluorescently labeled latex beads (L4530, Sigma Aldrich; final concentration of approximately $1 / 100$ in $1 / 3 \mathrm{X}$ FSW) or FITClabeled $E$. coli (Invitrogen E-2861; $2 \mathrm{mg} / \mathrm{ml}$ in 1/3X FSW). Excess particles were removed with three washes (15 mins each) in 1/3X FSW before tissues were fixed (as above) and cleared for imaging. All fluorescence imaging was performed on a Zeiss LSM 710 confocal microscope.

\section{Electron microscopy}

For scanning electron microscopy (SEM), live nematosomes were removed from adult polyps and concentrated in a minimal amount $(\sim 300 \mu \mathrm{l})$ of $1 / 3 \mathrm{X}$ FSW which was pipetted directly onto a pre-wetted $0.2 \mu \mathrm{m}$ GTTP filter (Millipore GTTP01300) mounted in a Swinnex filter holder in preparation for fixation. For TEM, whole polyps or spawned egg masses (containing nematosomes) were immobilized in $7.14 \% \mathrm{MgCl}_{2}$ for $15 \mathrm{~min}$ at $25{ }^{\circ} \mathrm{C}$ and collected/fixed in $1.5 \mathrm{ml}$ sterile microcentrifuge tubes. All other fixation, preparation, and imaging methods were performed as previously described [42].

\section{Transcriptome sequencing and analysis}

For sequence analysis, live nematosomes were removed from approximately 150-200 adult $N$. vectensis by making a small incision in the body wall of the polyp (near the pharynx) and then squeezing the nematosomes out into the surrounding $1 / 3 \mathrm{X}$ FSW in a small glass dish. Liberated nematosomes were then washed extensively in several changes of $1 / 3 \mathrm{X}$ FSW, immobilized in $7.14 \%$ $\mathrm{MgCl}_{2}$ on ice for $15 \mathrm{~min}$, and pooled into a single sample to recover enough material for one RNA-Seq replicate. This process was repeated three separate times (total $\sim 500$ adult polyps) to generate enough material for three independent replicates. For comparative transcriptomic assays, we also collected mesenteries (two mesenteries each from three adult polyps were pooled into a single sample; a total of three such samples were 
collected for sequencing) and tentacles (total of 30 tentacles from three adults, pooled; repeated for a total of three replicates). It should be noted that nematosomes develop from the mesenteries and although no budding nematosomes were observed at the time of collection of this tissue, we cannot exclude the possibility that our mesentery samples also contain nearly mature nematosomes (just prior to budding). All tissue samples (nematosomes, mesenteries, and tentacles) were transferred (with minimal 1/3X FSW) to sterile $1.5 \mathrm{ml}$ microcentrifuge tubes before being snap-frozen on dry ice. Samples were stored at $-80{ }^{\circ} \mathrm{C}$ for two weeks prior to processing. RNA was extracted and using a combination of Tri-Reagent (Sigma T9424; manufacturer's protocol) and the RNAEasy Mini kit (Qiagen 74104; manufacturer's protocol) and purified RNA samples were shipped to sequencing facilities on dry ice. cDNA library preparation and sequencing were performed commercially by SeqWright DNA Technology Services (Houston, TX, USA; 1 replicate of each tissue) or by the University of Florida's Integrative Center for Biotechnology Research (2 replicates of each tissue).

Paired raw reads were trimmed for quality and to remove adapter sequences using Trimmomatic [52] and error-corrected using the ErrorCorrectReads.pl utility from AllPaths-LG [53]. (See Additional file 9 for bioinformatic commands.) Unpaired reads resulting from these processes were included with the left reads for subsequent assembly steps. Cleaned, error-corrected reads (paired and unpaired) were then assembled de novo using Trinity $[49,50]$. To identify potential foreign/ contaminating sequences, we performed an alien index analysis (https://github.com/josephryan/alien_index) following the methods of Gladyshev et al [85]. In brief, we downloaded whole proteome data from the UniProt website (http://www.uniprot.org/uniprot/; keyword 181 "proteome") for diverse taxa: metazoans (SwissProt taxonomy ID: 33208; augmented by inclusion of the following pivotal taxa from NCBI unless otherwise indicated: Amphimedon queenslandica, Hydra magnipapillata, Mnemiopsis leidyi, $N$. vectensis from two sources: http://genome.jgi.doe.gov/Nemve1/Nemve1.home.html and www.cnidariangenomes.org, Trichoplax adhaerens), non-metazoan eukaryotes (ID: 2759), archaea (ID: 2157) and bacteria (ID: 2). We evaluated our reference transcriptome against this database using blastx and then calculated the alien index (AI) for all sequences as the ratio of the top non-metazoan blastx hit to the top metazoan blastx hit. All sequences for which $\mathrm{AI}>0$ (i.e., with better BLAST hits in nonmetazoan taxa) were inspected individually to determine if they were part of the $N$. vectensis genome. Of these, only sequences with significant blastn hits in $N$. vectensis ( $>70 \%$ sequence identity for $\geq 200 \mathrm{bp}$ ) were retained for further analysis.
Differential expression was assayed using DESeq2 version 1.11.23 implemented in R 3.2.3 following the recommendations in the User's Guide [57]. DESeq2 uses a generalized linear model with negative binomial distribution and significance is assayed via the Wald test on normalized (to library size and sample variance) count data. Following significance testing, we excluded all data for which the false discovery rate (FDR/adjusted p-value) was $\geq 0.05$ and fold change $\left(\log _{2} \mathrm{FC}\right)$ was $<|2|$ in pairwise comparisons of any two tissues. Upregulated sequences were identified as those which were expressed significantly higher in the one tissue relative to both other tissues; uniquely expressed sequences were identified as the subset of upregulated sequences that were upregulated in a given tissue $(\mathrm{FDR}<0.05, \log \mathrm{FC}>2)$ and not expressed (average count across replicates within a tissue $<2$ ) in either other tissue. To confirm our differential expression analysis, we validated this approach using qPCR (below) on 20 randomly selected genes from the list of sequences upregulated in nematosomes. Upregulated sequences were functionally annotated against the NCBI Homo sapiens reference transcriptome (version $3 / 25 / 2015$ ) using the AnnotationFuncs package in Bioconductor for R [60].

To identify novel transcripts from our dataset, we used a stringent approach to assigning sequence orthology based on reciprocal blastp searches of an expansive metazoan database using OrthoMCL $[58,59]$. All 17,313 expressed transcripts with ORFs $>100$ aa were translated into all six reading frames (Transdecoder; http:// transdecoder.github.io/) and all resulting predicted proteins were searched against a reference database containing two different sources of $N$. vectensis data: the JGI database (http://genome.jgi.doe.gov/Nemve1/ Nemve1.home.html) and publicly available de novo transcriptome database from the Technau Lab at the University of Vienna (http://www.cnidariangenomes.org/). As there are currently no genomic data available for the two other species of Nematostella ( $N$. polaris and $N$. nathorstii), our use of the term "Nematostella-specific" refers to transcripts present in a representative of the genus Nematostella ( $N$. vectensis) and absent from a representative of the Edwarsiidae (E. lineata). This reference database also included transcriptome data from several other taxa (all available publicly, unless otherwise specified): anthozoan cnidarians - Acropora digitifera, Aiptasia pallida [86], Anthopleura elegantissima, Edwardsiella lineata, Fungia scutaria, and Nephthyigorgia sp. [86]; other cnidarians - Alatina alata [86], Atolla vanhoeffeni [86], Hydra magnipapillata, Podocoryna carnea [86]; noncnidarian taxa - Amphimedon queenslandica (Porifera), Branchiostoma floridae (Chordata), Capitella teleta (Annelida), Drosophila melanogaster (Arthropoda), Homo 
sapiens (Chordata), Mnemiopsis leidyi (Ctenophora), and Trichoplax adhaerens (Placozoa). Ortholog clustering was then assayed as follows: (i) Nematostella-specific sequences grouped only with other $N$. vectensis sequences from our reference transcriptome, the JGI database, and/ or the Technau database, (ii) Edwardsiidae-specific sequences grouped only with sequences from the $N$. vectensis databases and $E$. lineata, (iii) anthozoan-specific sequences grouped with sequences from $A$. digitifera, $A$. elegantissima, A. pallida, F. scutaria and/or Nephthyigorgia sp., (iv) cnidarian-specific sequences grouped with $A$. alata, A. vanhoeffeni, H. magnipapillata, and/or P. carnea sequences, and $(\mathrm{v})$ conserved/metazoan sequences grouped with sequences from any of the following taxa: A. queenslandica, B. floridanus, C. teleta, D. melanogaster, H. sapiens, M. leidyi, or T. adhaerens. Predicted proteins that resulted from alternative ORFs in the same transcript sequence were subjected to orthology clustering independently but the final orthology of the transcript was assigned based on the highest value of the resulting categories (i.e., if any of the alternative proteins grouped with a conserved/ metazoan sequence, the transcript was assigned to the conserved/metazoan group). (See Additional file 8 for a summary of the orthology and differential expression analyses.)

\section{Quantitative Real-Time PCR (qPCR) and standard PCR}

Nematosomes were isolated from freshly spawned egg masses (as above), rinsed 3-5 times in 1/3X FSW and pooled into one sample in a sterile $1.5 \mathrm{ml}$ centrifuge tube on ice; this was repeated three times. Three replicate samples consisting of all eight mesenteries from each of three adult polyps (nine polyps total) and three replicate samples of all sixteen tentacles from each of three adult polyps (same nine polyps used for mesentery collections) were also collected. All excised tissues were maintained on ice after dissection and processed within $1 \mathrm{~h}$ of removal from the polyp. Total RNA was extracted using Tri-Reagent and cDNA was synthesized from $1 \mu \mathrm{g}$ of total RNA using the Advantage 2 RT for PCR kit (Clontech 639506), as previously described [87]. All primers were designed using the Primer3 utility in Geneious v.7.1.8 ([88] and qPCR primer efficiency was tested in a five-point standard curve (see Additional file 8 for primer sequences). qPCR was performed on $10 \mu \mathrm{l}$ triplicates for each replicate sample using a Roche LightCycler 480 thermocycler and the manufacturer's recommended SYBR mix (Roche 04887352001). Analysis of melt curves ensured our samples were free from genomic contaminants. Raw data were averaged (across biological replicates), normalized to the expression of housekeeping gene ATP synthetase, and are presented relative to the expression of each (normalized) transcript in nematosomes (set to 0). ANOVA was performed, as above, using $\mathrm{R}$ [84] and data are presented as means \pm SD.
To further examine the specificity of the transcripts we identified as nematosome-specific with RNA-Seq and qPCR analyses, we attempted to amplify 10 nematosomespecific genes from three tissues not sampled for RNASeq - mixed-stage embryos (blastula through late planula), body wall, and pharynx - using standard PCR. Adult polyps were spawned and fertilized eggs were separated from egg jelly as above. Embryos were incubated at $16{ }^{\circ} \mathrm{C}$ for $48,72,96,120$, or $144 \mathrm{~h}$ to generate a sample of embryos from blastula, early planula, and late planula stages. Embryos were collected at the indicated stage, homogenized in Tri-Reagent by vigorous vortexing $\left(30 \mathrm{~s}\right.$ at $\left.25^{\circ} \mathrm{C}\right)$ and stored at $-80{ }^{\circ} \mathrm{C}$ until the remaining embryonic samples had been collected. Total RNA was extracted at the same time from all samples $24 \mathrm{~h}$ after the final collection of embryos and cDNA was synthesized (as above) immediately following RNA extraction. Body wall tissue was collected from adult polyps by bisecting animals at the aboral end of the pharynx and then freeing the body wall of the mesenteries as close to the point of insertion as possible. The entire aboral body wall was collected from three polyps and pooled into a single tube for RNA extraction and cDNA synthesis. Pharynx was collected from the same three individuals by removing the tentacles and hypostome at the oral end of the pharynx and freeing the pharynx from the surrounding body wall. Body wall and pharynx samples were stored on ice for no more than one hour after dissection and RNA extraction and cDNA synthesis were performed as described above. Ten sequences were randomly selected from the pool of nematosomespecific transcripts and primers were designed (as above) to amplify a $1000 \mathrm{bp}$ region of the transcript. Standard PCR was performed using taq polymerase (New England Biolabs M0267L) following the manufacturer's recommendations and amplification was performing for 35 cycles using an extension time of 1.5 min per cycle. PCR products were separated on a $1 \%$ agarose gel and visualized using SYBRsafe gel stain (Invitrogen S-33102).

\section{Additional files}

\footnotetext{
Additional file 1: Live nematosomes moving through the pharyngeal region of an adult polyp. (MP4 $17442 \mathrm{~kb}$ )

Additional file 2: (A) Small cellular material moving through the tentacle lumen of a primary/immature polyp. (B) Small cellular material and nematosomes moving through the tentacle of an adult polyp. (ZIP 51824 kb)

Additional file 3: Further examination of nematosomes. (A) Proliferating cells in an individual nematosome labeled while it was circulating through the body cavity (red - proliferative nuclei, blue - non-proliferative nuclei, $1 \mu \mathrm{M}$ DAPI). A total of three nematosomes have ever been observed to have proliferating cells via EdU. (B) (C-E) PCR confirms that nematosome-specific transcripts are uniquely expressed in nematosomes. Nine out of ten primers designed to amplify transcripts expressed uniquely in nematosomes failed to amplify products of the predicted size in CDNA samples extracted from mixed stage embryos, adult pharynx, and adult body wall. The product amplified using primers for Nv5804
} 
(lane 1) appears to be ubiquitously expressed across all three tissues assayed. Actin was amplified as a positive control in lane 11. Faint bands in lanes 7-9 of the pharynx are of the wrong size and likely represent mis-priming. Lane 1: Nv5804, 2: Nv4198, 3: Nv13913, 4: Nv18469, 5: Nv5803, 6: Nv5749, 7: Nv9367, 8: Nv19938, 9: Nv18000, 10: Nv2134, 11 actin. See Additional file 8 for primer sequences. (PNG $1597 \mathrm{~kb}$ )

Additional file 4: Live nematosomes can subdue recently hatched brine shrimp (Artemia salina). (MP4 $49604 \mathrm{~kb}$ )

Additional file 5: Cnidocytes in the tentacle tips of an adult polyp can subdue A. salina. (MP4 $48606 \mathrm{~kb}$ )

Additional file 6: 1/3X FSW alone (without nematosomes) has no effect on A. salina. (MP4 $71560 \mathrm{~kb}$ )

Additional file 7: Nucleotide sequences for 82 sequences with alien index $>0$. (FA $51 \mathrm{~kb}$ )

Additional file 8: Summary of everything. Sheet 1: Stress Gene Survey. List of tissue-specific targets and their reciprocal best blast hit (accession ID) from the dataset presented by Reitzel et al [63]. Included fields: Tissue, unique transcript ID (NvecRef ID), reciprocal best blastx hit (Hit), stressor type (pathogen, wound, or chemical). Transcripts with reciprocal best blast hits involved in both pathogen and wound stress are indicated as: Pathogen.Wound. Sheet 2: Transcriptome Summary. Summary of all 17,313 transcripts used for differential expression and orthology analyses. Each entry contains the following: unique transcriptome ID (NvecRef ID), transcript length (in nucleotides), orthology group ID (GrID): N - Nematostella-specific, E - Edwarsiidae-specific, A - anthozoan-specific, C cnidarian-specific, M - conserved/metazoan, number of tissues (out of three) in which the transcript is expressed, differential expression result (UP/mes is upregulated in the mesenteries relative to both other tissues), mean counts across all tissues (AllMean) and across replicates within a tissue (MesMean, NemMean, TenMean), log2 fold change for all pairwise tissue comparisons (negative values indicate upregulation in the second tissue), adjusted p-values/false discovery rates for all pairwise comparisons, and raw counts for each tissue replicate. Sheet 3: Alien Index Results. Fields: unique transcriptome ID (NvecRef ID), alien index (Al), \% identity and coverage of the top blast hit, and whether the sequence was deemed present in the genome (http://genome.jgi.doe.gov/Nemve1/Nemve1.home.html). Sheet 4: Primers. Sequences for all primers used in GPCR and standard PCR analyses. Primer sequences for each transcript are presented $5^{\prime} \rightarrow 3^{\prime}$. (XLSX $\left.4037 \mathrm{~kb}\right)$

Additional file 9: Commands for bioinformatic analyses and statistical methods. (TXT $5 \mathrm{~kb}$ )

Additional file 10: Full $G O$ annotation reports for upregulated transcripts by tissue. Sheet 1 (summary) includes full Blast2GO reports for all three tissues. Abbreviations: Mes - mesenteries, Nem - nematosomes, Ten - tentacles, Gene Symbol - NCBI approved gene symbol, GO ID gene ontology identifier, BP (GO category) - Biological process, CC cellular component, MF - molecular function, TrID - transcript ID (NvecRef transcriptome), HumRef - accession number (human orthologs) for NCBI's Refseq database. (XLSX $183 \mathrm{~kb}$ )

Additional file 11: Live nematosomes are still present (and moving) in the degenerating egg jelly after most of the $\mathrm{N}$. vectensis planulae have emerged. (MP4 $88967 \mathrm{~kb}$ )

\footnotetext{
Abbreviations

1/3X FSW: 1/3X filtered seawater; ANCOVA: analysis of covariance; ANOVA: analysis of variance; BLAST: basic local alignment search tool; CEGMA: Core Eukaryotic Genes Mapping Approach; DAPI: 2-(4amidinophenyl)-1H-indole-6-carboxamidine; DIC: differential interference contrast (microscopy); FITC: fluorescein isothiocyanate; GO: gene ontology; NCBI: National Center for Biotechnology Information; ORF: Open Reding Frame; PCR: polymerase chain reaction; PTw: phosphate buffered saline with $0.1 \%$ Tween-20; PTx: phosphate buffered saline with $0.1 \%$ Triton-X; qPCR: quantitative Real Time PCR; RNA-Seq: RNA sequencing; SD: standard deviation; SEM: scanning electron microscopy; TEM: transmission electron microscopy.
}

\section{Acknowledgements}

The authors would like to thank Tina Carvahlo and Marilyn Dunlap in the Biological Electron Microscopy Facility at the University of Hawaii and C. Titus Brown and his laboratory/associates involved in the NGS course at Kellogg Biological Station for their help interpreting our data. The anti-mcol1 and anti-mcol3 antibodies were a generous gift from the laboratory of Dr. Suat Ozbek. This work was supported by the National Aeronautics and Space Administration (NNX14AG70G).

\section{Authors' contributions}

LSB conceived of the study, collected tissues, carried out all laboratory work, conducted bioinformatic analyses, and wrote the manuscript. MQM participated in the design and coordination of the study, collected tissues, and evaluated multiple drafts of the manuscript. JFR participated in the design and coordination of the study, conducted bioinformatic analyses, and evaluated multiple drafts of the manuscript. All authors read and approved the final manuscript.

\section{Availability of data and materials}

Raw sequencing reads and the three reference transcriptomes assembled from them are available in the European Nucleotide Archive, Project Accession: PRJEB13676 (http://www.ebi.ac.uk/ena/data/view/PRJEB13676), unique sample identifiers: mesenteries: SAMEA3937624, SAMEA3937625, SAMEA3937626; nematosomes: SAMEA3937621, SAMEA3937622, SAMEA3937623; tentacles: SAMEA3937635, SAMEA3937636, SAMEA3937637. The remaining datasets supporting the conclusions of this article are included within the article (and its additional files).

\section{Ethics}

No human subjects were used in this study and the invertebrate animals used in this study are not subject to regulation by animal ethics committees.

\section{Consent}

All authors have approved this manuscript for publication.

\section{Competing interests}

The authors declare that they have no competing interests.

\section{Author details}

'Whitney Laboratory for Marine Bioscience, University of Florida, 9505 Ocean Shore Blvd, St. Augustine, FL 32080, USA. ${ }^{2}$ Department of Biology, University of Florida, Gainesville, FL 32611, USA.

Received: 27 April 2016 Accepted: 12 May 2016

Published online: 23 May 2016

\section{References}

1. Neme R, Tautz D. Phylogenetic patterns of emergence of new genes support a model of frequent de novo evolution. BMC Genomics. 2013;14:117.

2. Ding $Y$, Zhou Q, Wang W. Origins of new genes and evolution of their novel functions. Annu Rev Ecol Evol Syst. 2012;43(1):345-63.

3. Prud'homme B, Gompel N, Carroll SB. Emerging principles of regulatory evolution. Proc Natl Acad Sci U S A. 2007;104 Suppl 1:8605-12.

4. Cheatle Jarvela AM, Hinman VF. Evolution of transcription factor function as a mechanism for changing metazoan developmental gene regulatory networks. EvoDevo. 2015;6(1):3.

5. Tautz D, Domazet-Loso T. The evolutionary origin of orphan genes. Nat Rev Genet. 2011;12(10):692-702.

6. Khalturin K, Hemmrich G, Fraune S, Augustin R, Bosch TC. More than just orphans: are taxonomically-restricted genes important in evolution? Trends Genet. 2009;25(9):404-13.

7. Foret S, Knack B, Houliston E, Momose T, Manuel M, Queinnec E, Hayward DC, Ball EE, Miller DJ. New tricks with old genes: the genetic bases of novel cnidarian traits. Trends Genet. 2010;26(4):154-8.

8. Chen SD, Zhang YE, Long MY. New genes in Drosophila quickly become essential. Science. 2010;330(6011):1682-5.

9. Hwang JS, Takaku Y, Momose T, Adamczyk P, Ozbek S, Ikeo K, Khalturin K, Hemmrich G, Bosch TC, Holstein TW et al. Nematogalectin, a nematocyst protein with GlyXY and galectin domains, demonstrates nematocytespecific alternative splicing in Hydra. Proc Natl Acad Sci U S A. 2010; 107(43):18539-44 
10. Khalturin K, Anton-Erxleben F, Sassmann S, Wittlieb J, Hemmrich G, Bosch TC. A novel gene family controls species-specific morphological traits in Hydra. PLoS Biol. 2008;6(11):e278.

11. Milde S, Hemmrich G, Anton-Erxleben F, Khalturin K, Wittlieb J, Bosch TC. Characterization of taxonomically restricted genes in a phylum-restricted cell type. Genome Biol. 2009;10(1):R8.

12. Jasper WC, Linksvayer TA, Atallah J, Friedman D, Chiu JC, Johnson BR. Large-scale coding sequence change underlies the evolution of postdevelopmental novelty in honey bees. Mol Biol Evol. 2015;32(2):334-46.

13. Mikheyev AS, Linksvayer TA. Genes associated with ant social behavior show distinct transcriptional and evolutionary patterns. eLife. 2015;4:e04775.

14. Brigandt I, Love AC. Conceptualizing evolutionary novelty: moving beyond definitional debates. J Exp Zool B Mol Dev Evol. 2012;318(6):417-27.

15. Peterson T, Muller GB. What is evolutionary novelty? Process versus character based definitions. J Exp Zool B Mol Dev Evol. 2013;320(6):345-50.

16. Hwang JS, Ohyanagi H, Hayakawa S, Osato N, Nishimiya-Fujisawa C, Ikeo K David CN, Fujisawa T, Gojobori T. The evolutionary emergence of cell typespecific genes inferred from the gene expression analysis of Hydra. Proc Natl Acad Sci U S A. 2007;104(37):14735-40.

17. Yang L, Zou M, Fu B, He S. Genome-wide identification, characterization, and expression analysis of lineage-specific genes within zebrafish. BMC Genomics. 2013;14:65.

18. Ostman C. A guideline to nematocyst nomenclature and classification, and some notes on the systematic value of nematocysts. Scientia Marina. 2000; 64:31-46.

19. Fautin D, Mariscal RN. Cnidaria: Anthozoa. In: Harrison FW, Westfall J, editors. Microscopic Anatomy of Invertebrates. Chichester: Alan R. Liss; 1991. p. 267-358.

20. Won JH, Rho BJ, Song J. A phylogenetic study of the Anthozoa (phylum Cnidaria) based on morphological and molecular characters. Coral Reefs. 2001;20(1):39-50.

21. Schlesinger A, Zlotkin E, Kramarsky-Winter E, Loya Y. Cnidarian internal stinging mechanism. P Roy Soc B-Biol Sci. 2009;276(1659):1063-7.

22. Rodriguez E, Barbeitos M, Daly M, Gusmao LC, Haussermann V. Toward a natural classification: phylogeny of acontiate sea anemones (Cnidaria, Anthozoa, Actiniaria). Cladistics. 2012;28(4):375-92.

23. Östman C, Roat Kultima J, Roat C, Rundblom K. Acontia and mesentery nematocysts of the sea anemone Metridium senile (Linnaeus, 1761) (Cnidaria: Anthozoa). Scientia Marina. 2010;74(3):483-97.

24. Darling JA, Reitzel AR, Burton PM, Mazza ME, Ryan JF, Sullivan JC, Finnerty JR. Rising starlet: the starlet sea anemone, Nematostella vectensis. Bioessays 2005;27(2):211-21.

25. Stephenson TA. The British Sea Anemones, vol. II. London: The Ray Society; 1935.

26. Crowell S. A new sea anemone from Woods Hole, Massachusetts. J Wash Acad Sci. 1946;36:57-60.

27. Robson E. A sea-anemone from brackish water. Nature. 1957;179:787-8.

28. Carlgren O. Actiniaria, Part I. Danish Ingolf Expedition. 1921;V:1-241.

29. Carlgren O. Actiniaria, zoantharia and madreporaria. Zool Iceland. 1939; 2(8):1-20.

30. Carlgren O. A survey of the ptychodactiaria, corallimorpharia and actiniaria. K Svenska Vetensk Akad Handl. 1949;1(1):1-121.

31. Williams RB. A redescription of the brackish-water sea anemone Nematostella vectensis Stephenson, with an appraisal of congeneric species. J Nat Hist. 1975; 9(1):51-64.

32. Hand C. Another sea anemone from California and the types of certain California species. J Wash Acad Sci. 1957;47:411-4.

33. Williams RB. Studies on the nematosomes of Nematostella vectensis Stephenson (Coelenterata: Actiniaria). J Nat Hist. 1979;13(1):69-80.

34. Frank P, Bleakney JS. Histology and sexual reproduction of anemone Nematostella vectensis Stephenson 1935. J Nat Hist. 1976;10(4):441-9.

35. Hand C, Uhlinger KR. The culture, sexual and asexual reproduction, and growth of the sea-anemone Nematostella vectensis. Biol Bull-Us. 1992; 182(2):169-76.

36. Wagner GP, Lynch VJ. Evolutionary novelties. Curr Biol. 2010;20(2):R48-52.

37. Hall BK, Kerney R. Levels of biological organization and the origin of novelty. J Exp Zool B Mol Dev Evol. 2012;318(6):428-37.

38. Zenkert C, Takahashi T, Diesner MO, Ozbek S. Morphological and molecular analysis of the Nematostella vectensis cnidom. PLoS One. 2011;6(7):e22725.

39. Babonis LS, Martindale MQ. Old cell, new trick? Cnidocytes as a model for the evolution of novelty. Integr Comp Biol. 2014;54(4):714-22.
40. Tang PC, Watson GM. Cadherin-23 may be dynamic in hair bundles of the model sea anemone Nematostella vectensis. PLoS One. 2014;9(1):e86084.

41. Watson GM, Mire P, Kinler KM. Mechanosensitivity in the model sea anemone Nematostella vectensis. Mar Biol. 2009;156(10):2129-37.

42. DuBuc TQ, Dattoli AA, Babonis LS, Salinas-Saavedra M, Rottinger E, Martindale $M Q$, Postma M. In vivo imaging of Nematostella vectensis embryogenesis and late development using fluorescent probes. BMC Cell Biol. 2014;15:44.

43. Westfall JA, Sayyar KL, Elliott CF. Cellular origins of kinocilia, stereocilia, and microvilli on tentacles of sea anemones of the genus Calliactis (Cnidaria : Anthozoa). Invert Biol. 1998;117(3):186-93.

44. Watson GM, Hessinger DA. Cnidocytes and adjacent supporting cells form receptor-effector complexes in anemone tentacles. Tissue Cell. 1989;21(1):17-24.

45. Watson GM, Hessinger DA. Receptor-mediated endocytosis of a chemoreceptor involved in triggering the discharge of Cnidae in a sea-anemone tentacle. Tissue Cell. 1987:19(6):747-55.

46. Putnam NH, Srivastava M, Hellsten U, Dirks B, Chapman J, Salamov A, Terry A Shapiro $H$, Lindquist $E$, Kapitonov $W$ et al. Sea anemone genome reveals ancestral eumetazoan gene repertoire and genomic organization. Science. 2007:317(5834):86-94.

47. Helm RR, Siebert S, Tulin S, Smith J, Dunn CW. Characterization of differential transcript abundance through time during Nematostella vectensis development. BMC Genomics. 2013;14:266.

48. Tulin S, Aguiar D, Istrail S, Smith J. A quantitative reference transcriptome for Nematostella vectensis early embryonic development: a pipeline for de novo assembly in emerging model systems. EvoDevo. 2013:4:16.

49. Grabherr MG, Haas BJ, Yassour M, Levin JZ, Thompson DA, Amit I, Adiconis X, Fan L, Raychowdhury R, Zeng Q, et al. Full-length transcriptome assembly from RNA-Seq data without a reference genome. Nat Biotechnol. 2011;29(7):644-52

50. Haas BJ, Papanicolaou A, Yassour M, Grabherr M, Blood PD, Bowden J, Couger MB, Eccles D, Li B, Lieber M, et al. De novo transcript sequence reconstruction from RNA-seq using the Trinity platform for reference generation and analysis. Nat Protoc. 2013;8(8):1494-512.

51. Tasnim M, Ma SN, Yang EW, Jiang T, Li W. Accurate inference of isoforms from multiple sample RNA-Seq data. BMC Genomics. 2015;16 Suppl 2:S15.

52. Bolger AM, Lohse M, Usadel B. Trimmomatic: a flexible trimmer for Illumina sequence data. Bioinformatics. 2014;30(15):2114-20.

53. Gnerre S, Maccallum I, Przybylski D, Ribeiro FJ, Burton JN, Walker BJ, Sharpe T, Hall G, Shea TP, Sykes S, et al. High-quality draft assemblies of mammalian genomes from massively parallel sequence data. Proc Natl Acad Sci U S A. 2011;108(4):1513-8.

54. Langmead B, Trapnell C, Pop M, Salzberg SL. Ultrafast and memory-efficient alignment of short DNA sequences to the human genome. Genome Biol. 2009;10(3):R25.

55. Quinlan AR, Hall IM. BEDTools: a flexible suite of utilities for comparing genomic features. Bioinformatics. 2010;26(6):841-2.

56. Parra G, Bradnam K, Korf I. CEGMA: a pipeline to accurately annotate core genes in eukaryotic genornes. Bioinformatics. 2007;23(9):1061-7.

57. Love Ml, Huber W, Anders S. Moderated estimation of fold change and dispersion for RNA-seq data with DESeq2. Genome Biol. 2014;15(12):550.

58. Li L, Stoeckert CJ, Roos DS. OrthoMCL: Identification of ortholog groups for eukaryotic genomes. Genome Res. 2003;13(9):2178-89.

59. Enright AJ, Van Dongen S, Ouzounis CA. An efficient algorithm for largescale detection of protein families. Nucleic Acids Res. 2002;30(7):1575-84.

60. Edwards SM. AnnotationFuncs: Annotation translation functions for Bioconductors annotation packages. 2011. In. http://www.iysik.com/r/annotationfuncs. Accessed 9 March 2016.

61. Ricciotti E, FitzGerald GA. Prostaglandins and Inflammation. Arterioscl Throm Vas. 2011;31(5):986-1000

62. Capdevila JH, Falck JR. The CYPP450 arachidonic acid monooxygenases: From cell signaling to blood pressure regulation. Biochem Biophys Res Commun. 2001;285(3):571-6.

63. Reitzel AM, Sullivan JC, Traylor-Knowles N, Finnerty JR. Genomic survey of candidate stress-response genes in the estuarine anemone Nematostella vectensis. Biol Bull-Us. 2008;214(3):233-54.

64. Moran Y, Genikhovich G, Gordon D, Wienkoop S, Zenkert C, Ozbek S, Technau U, Gurevitz M. Neurotoxin localization to ectodermal gland cells uncovers an alternative mechanism of venom delivery in sea anemones. Proc Biol Sci. 2012;279(1732):1351-8.

65. Pantin CFA. Excitation of nematocysts. Nature. 1942;149:109-9. 
66. Watson GM, Hessinger DA. Cnidocyte mechanoreceptors are tuned to the movements of swimming prey by chemoreceptors. Science. 1989; 243(4898):1589-91.

67. Westfall JA, Landers DD, McCallum JD. Different nematocytes have different synapses in the sea anemone Aiptasia pallida (Cnidaria, anthozoa). J Morphol. 1998;238(1):53-62.

68. Westfall JA, Landers DD, Mccallum JD. Ultrastructure of neuro-spirocyte synapses in the sea anemone Aiptasia pallida (Cnidaria, Anthozoa, Zoantharia). J Morphol. 1999;241(2):165-73.

69. Peteya DJ. Ciliary-cone sensory cell of anemones and cerianthids. Tissue Cell. 1975;7(2):243-52.

70. Thorington GU, Hessinger DA. Control of Cnida discharge.3. Spirocysts are regulated by 3 classes of chemoreceptors. Biol Bull-Us. 1990;178(1):74-83.

71. Metchnikoff E. Lecons sur la Pathologie Comparee de Inflammation. Paris: Masson; 1892.

72. Olano CT, Bigger CH. Phagocytic activities of the gorgonian coral Swiftia exserta. J Invertebr Pathol. 2000;76(3):176-84

73. Patterson MJ, Landolt ML. Cellular reaction to injury in the anthozoan Anthopleura elegantissima. J Invertebr Pathol. 1979;33(2):189-96.

74. Devuyst O, Dahan K, Pirson Y. Tamm-Horsfall protein or uromodulin: new ideas about an old molecule. Nephrol Dial Transpl. 2005;20(7):1290-4.

75. El-Achkar TM, McCracken R, Liu Y, Heitmeier MR, Bourgeois S, Ryerse J, Wu XR. Tamm-Horsfall protein translocates to the basolateral domain of thick ascending limbs, interstitium, and circulation during recovery from acute kidney injury. Am J Physiol-Renal. 2013;304(8):F1066-75.

76. DuBuc TQ, Traylor-Knowles N, Martindale MQ. Initiating a regenerative response; cellular and molecular features of wound healing in the cnidarian Nematostella vectensis. BMC Biol. 2014;12:24.

77. Johnson BR, Tsutsui ND. Taxonomically restricted genes are associated with the evolution of sociality in the honey bee. BMC Genomics. 2011;12:164.

78. Lobo D, Vico FJ. Evolution of form and function in a model of differentiated multicellular organisms with gene regulatory networks. Biosystems. 2010; 102(2-3):112-23.

79. Bosch TC. Rethinking the role of immunity: lessons from Hydra. Trends Immunol. 2014:35(10):495-502.

80. David CN, Ozbek S, Adamczyk P, Meier S, Pauly B, Chapman J, Hwang JS, Gojobori T, Holstein TW. Evolution of complex structures: minicollagens shape the cnidarian nematocyst. Trends Genet. 2008;24(9):431-8.

81. Hoekstra HE, Coyne JA. The locus of evolution: Evo devo and the genetics of adaptation. Evolution. 2007;61(5):995-1016.

82. Fritzenwanker $\mathrm{JH}$, Technau $\mathrm{U}$. Induction of gametogenesis in the basal cnidarian Nematostella vectensis(Anthozoa). Dev Genes Evol. 2002;212(2):99-103.

83. Szczepanek S, Cikala M, David CN. Poly-gamma-glutamate synthesis during formation of nematocyst capsules in Hydra. J Cell Sci. 2002;115(4):745-51.

84. R Development Core Team. R: A language and environment for statistical computing. Vienna: R Foundation for Statistical Computing; 2008.

85. Gladyshev EA, Meselson M, Arkhipova IR. Massive horizontal gene transfer in bdelloid rotifers. Science. 2008;320(5880):1210-3.

86. Zapata F, Goetz FE, Smith SA, Howison M, Siebert S, Church SH, Sanders SM, Ames CL, McFadden CS, France SC, et al. Phylogenomic analyses support traditional relationships within Cnidaria. PloS One. 2015;10(10):e0139068.

87. Rottinger $\mathrm{E}$, Dahlin $\mathrm{P}$, Martindale MQ. A framework for the establishment of a cnidarian gene regulatory network for "endomesoderm" specification: the inputs of $\beta$-catenin/TCF signaling. PLoS Genet. 2012;8(12):e1003164.

88. Kearse M, Moir R, Wilson A, Stones-Havas S, Cheung M, Sturrock S, Buxton S, Cooper A, Markowitz S, Duran C, et al. Geneious basic: an integrated and extendable desktop software platform for the organization and analysis of sequence data. Bioinformatics. 2012;28(12):1647-9.

\section{Submit your next manuscript to BioMed Central and we will help you at every step:}

- We accept pre-submission inquiries

- Our selector tool helps you to find the most relevant journal

- We provide round the clock customer support

- Convenient online submission

- Thorough peer review

- Inclusion in PubMed and all major indexing services

- Maximum visibility for your research

Submit your manuscript at www.biomedcentral.com/submit
Biomed Central 